\title{
Extensions and limitations of analytical airfoil broadband noise models
}

\author{
Michel Roger ${ }^{\circ}$ \& Stéphane Moreau* \\ 'École Centrale de Lyon \\ Laboratoire de Mécanique des Fluides et Acoustique \\ UMR CNRS 5509, 36 avenue Guy de Collongue \\ 69134 ECULLY Cedex, FRANCE \\ *GAUS, Sherbrooke University \\ Sherbrooke \\ Québec, CANADA
}

Received July 24, 2009; Revised January 20, 2010; Accepted February 2, 2010

\begin{abstract}
The present paper is a state-of-the-art of a special class of analytical models to predict the broadband noise generated by thin airfoils in a flow, either clean or disturbed. Three generating mechanisms are addressed, namely the noise from the impingement of upstream turbulence called turbulence-interaction noise, the noise due to the scattering of boundary-layer turbulence as sound at the trailing edge for an attached flow called trailing-edge noise, and the noise generated due to the formation of a coherent vortex shedding in the near wake of a thick trailing edge, called vortex-shedding noise. Different analytical models previously proposed for each mechanism are reviewed, as declinations of the same basic approach inherited from the pioneer work performed by Amiet in the seventies and based on an extensive use of Schwarzschild's technique. This choice is only an alternative to other models available in the literature and is made here for the sake of a unified approach. Issues dealing with the input data and related to the practical applications to fan noise predictions are rapidly outlined. The validity of the models is ckeched against dedicated experiments with thin airfoils and the limitations as the real configurations depart from the model assumptions are pointed out.
\end{abstract}

\section{INTRODUCTION}

Broadband noise prediction tools are a crucial need for low-noise designs in many rotating blade technologies, such as cooling fans, wind turbines and turbofan engine blade rows. However meeting this need by modern numerical simulations remains unrealistic at a reasonable cost in an industrial context. For a short-term preliminary design, simplified thus much faster and much less expensive predictions can be achieved by means of analytical techniques which provide closed-form expressions of the sound field. But this low-cost noise evaluation is at the price of drastic assumptions 
which possibly lead to substantial errors, and a careful assessment of the techniques must be made, for instance by comparing the results to dedicated laboratory experiments. Essentially a blade section or an airfoil is assimilated in the model to an unloaded rigid plate with zero thickness and camber, embedded in a uniform mean flow. The unsteady aerodynamic response to small disturbances is first calculated by linearized theories, inherited from pioneer works developed in the seventies. The sound field is calculated in a second step from the unsteady lift induced on the plate according to the acoustic analogy [1]. Both steps are achieved in the frequency domain. The sound field is calculated via a statistical analysis which is reliable only when the incriminated turbulence is close enough to an homogeneous and stationary random process.

The paper is reviewing some analytical models adapted or extended from the existing literature by the authors, and dealing with three major broadband noise mechanisms of an isolated airfoil, namely the impingement of upstream turbulence on a leading edge (turbulence-interaction noise), the scattering of boundary-layer turbulence as sound at a trailing edge (trailing-edge noise), and the vortex shedding in the near wake of a blunt trailing edge (vortex shedding noise). The input data are the statistics of the incident velocity, wall pressure or wake velocity upwash, respectively. The models are based on extended Amiet's isolated-airfoil response functions which account for non-compactness and fluid compressibility. Even though relevant, alternative models available in the literature (for instance detailed in [2, 3, 4, 5] among others) are not considered in this paper. The focus on Amiet's methodology is justified by the intention to address different mechanisms with the same mathematical background, for the sake of a somewhat unified analysis. The isolated-airfoil approach discussed in this paper is well suited for rotors with few blades and/or low solidity. Turbomachinery blade rows with a large number of blades and high solidity are better addressed using cascade response functions which account for the effect of adjacent blades on the response of a reference blade. This topic is not considered here and is covered by a specific literature (for instance based on Glegg's formulation [6], see ref. [7] for an indicative review). Moreover the theory addresses slightly loaded airfoils according to usual assumptions made in linearized aerodynamics of thin blades. The restriction includes small camber, thickness and angle of attack, as well as small fluctuations. Typically the set of assumptions remains reasonable for turbulent rates in the flow lower than $10 \%$, relative thicknesses of a couple of percent, and moderate cambers. Some of these aspects will be quantified later on. A priori only the blades of fans and compressors enter the validity domain, whereas highly cambered and thick blades found in turbines and similar applications should require numerical means. However, as shown in some applications mentioned in the paper, the linearized theory sometimes accomodates for values of some parameters such as the angle of attack and the relative thickness which are far from small.

The section 2 below is dealing with the mathematical statement of Schwarzschild's technique used as a basis for all subsequent developments. The different formulations and key assumptions are reminded for each investigated mechanism and the closed-form solutions are simply listed, reproduced from the reference papers. The experimental data base used for validation purposes is next decribed in section 3, separately for each 
mechanism. The limitations of the models are identified by comparing the predictions to the measurements, in both dimensional values and non-dimensional parameters more suited to the tracking of scaling laws. Finally different kinds of extensions which possibly apply to all three mechanisms are shortly outlined in section 4 .

\section{THEORETICAL BACKGROUND}

\subsection{Schwarzschild-based analytical formulations}

Broadband noise generation arises as turbulent structures experience rapid modifications, part of the inertia of the vortical motion being converted into sound due to fluid compressibility. In subsonic flows this conversion is more efficient when the modifications are due to the interaction with a solid surface, and more specifically when it occurs close to a geometrical singularity of the surface. The generating mechanisms addressed in this paper are special cases where the surface is modelled as a rigid flat plate with zero thickness and camber, and the singular points are either leading edges or trailing edges. Turbulenceinteraction noise results from the direct impingement of upstream turbulence at a leading edge, the vortical motion having suddenly its disturbance component perpendicular to the surface forced to zero. In contrast, trailing-edge noise is produced because the boundarylayer eddies suddenly experience the suppression of a bounding wall; secondary vorticity is then shed in the wake according to a full or partial Kutta condition. Vortex-shedding noise is due to the formation of vortices in the near wake of a trailing edge in a flow free of vortices just upstream of that edge. From a local point of view, all mechanisms involve a change in the boundary conditions applied to a vortical field, with different uniform conditions on each side of the incriminated edge. This suggests to formulate the mathematical problem as a matching between two sub-spaces, arguing that the effective length of the plate is large when compared to the size of the vortical eddies, and to eventually add corrections if needed. The basis for such a general approach is provided by the Schwarzschild's technique discussed in the paper and introduced in electromagnetism for the scattering of a wave by the edge of a semi-infinite plate. The technique is described in the book by Landahl [8]. It was first applied in aeroacoustics by Amiet in the seventies, dealing with turbulence-interaction noise $[9,10]$. It has been next extended to trailing-edge noise $[11,12]$. Its use for vortex-shedding noise has been proposed recently as a straightforward adaptation [13]. Essentially the three mechanisms are seen as wave scattering problems, formulated on the additional potential disturbance field introduced by the airfoil singularity in the incident (vortical) disturbed flow. For this potential field, the same generic theorem generates different closed-form solutions.

Schwarzschild's theorem is stated as follows. Let $\Phi$ be a two-dimensional (2D) scalar field solution of the Helmholtz equation and associated boundary conditions

$$
\begin{array}{ll}
\frac{\partial^{2} \Phi}{\partial x^{2}}+\frac{\partial^{2} \Phi}{\partial z^{2}}+\mu^{2} \Phi=0 \\
\Phi(x, 0)=f(x) & x \geq 0 \\
\frac{\partial \Phi}{\partial z}(x, 0)=0 & x<0
\end{array}
$$


where $f$ is a known function. This problem will be denoted by $\left(\mathcal{P}_{0}\right)$. Then for any $x<0$

$$
\Phi(x, 0)=\frac{1}{\pi} \int_{0}^{\infty} G(x, \xi, 0) f(\xi) d \xi
$$

with

$$
G(x, \xi, 0)=\sqrt{\frac{-x}{\xi}} \frac{e^{-i \mu(\xi-x)}}{\xi-x}
$$

Theories based on this theorem do not directly predict the whole field but only its trace distributed along the sub-space $(z=0, x<0)$ when the field is known along $(z=0$, $x>0)$ and its normal derivative is zero along $(z=0, x<0)$.

Now even the less sophisticated mathematical statements of airfoil noise problems are at least dealing with flat plates of finite chord length radiating in a three-dimensional (3D) space in the presence of a uniform flow. The flow is parallel to the chord, thus normal to the spanwise direction, and the span is assumed of infinite extent. Source coordinates are introduced as $\left(y_{1}, y_{2}\right)$ in the streamwise and spanwise directions respectively, with reference either at the leading edge or at the trailing edge. Another set of coordinates $\left(x_{1}, x_{2}, x_{3}\right)$ is introduced for the observer with reference at the airfoil center point. The chosen acoustic variable is solution of the convected Helmholtz equation in $3 \mathrm{D}$. The idea is to perform transformations in order to recover the aforementioned 2D canonical Schwarzschild's problem on the transformed variables, $(x, z)$ being obtained from $\left(y_{1}, y_{3}\right)$. This is first achieved by expanding the fluctuating quantities in Fourier components of wavenumbers $k_{2}$ in the spanwise direction, taking advantage of the infinite span. Each Fourier component, called a gust, is solution of a modified $2 \mathrm{D}$ convected Helmholtz equation. The problem $\left(\mathcal{P}_{0}\right)$ is found formally by a further transformation reducing the convected Helmholtz operator to a stationarymedium one. Once derived in its reduced form, the solution is transposed to the original variables by applying the inverse transformations. Finally the principle of the statistical approach will be to sum up all gust contributions to the radiated field and to take an ensemble average. The chosen field variable is either the disturbance pressure or the velocity potential, depending on the physical nature of the boundary conditions. Both are compatible with the rigidity condition since their normal derivatives have to be zero on the surface.

The remaining issue is that the actual chord length is finite. Though Schwarzschild's technique used as such would provide a consistent solution from the point of view of the edge more directly involved in the vortex dynamics of interest, the scattering effect of the complementary edge would be ignored. This effect can be accounted for by an iterative procedure. For a primary interaction taking place at one edge, a first-order field is derived assuming a virtual plate extending to infinity in the opposite direction. A second-order correction is then calculated to cancel the error made in the first-order one beyond the opposite edge. This correction is derived assuming that the plate is now infinite from the opposite edge. Higher-order iterative corrections are needed in 
principle and the procedure is expected to converge rapidly to the wanted solution of the problem with a finite chord. This is ensured in practice if the frequency is high enough.

The trace determined by Schwarzschild's technique is not enough for acoustic purposes. It only provides, directly or indirectly, the pressure jump along the airfoil, acting as the equivalent source distribution. In a second step the acoustic field is calculated away from the sources by a classical radiation integral, according to Ffowcs Williams \& Hawkings' acoustic analogy [1]. More precisely the sources are exactly the dipole contribution of the analogy, recognized as dominant at subsonic Mach numbers. Since the problem is solved in a non-compact and compressible context, the analogy is applied in its original form, based on the free-space Green's function, and implicitly accounts for the diffraction by the surface. If the iterative procedure is converged, the solution is exact in the sense that the results could be reproduced by a numerical simulation or an alternative theory addressing the same geometry and the same model problem. This has been shown numerically for the problem of the noise generated by an incident gust impinging on a flat plate, in a paper by Lockard \& Morris [14] where a semi-analytic solution equivalent to Amiet's formulation is used for comparisons. By the way, using Schwarzschild's technique is equivalent to solve Lighthill's equation with the Green's function tailored to the flat plate $[2,5]$, and is equivalent to alternative approches such as developed by Howe for trailing-edge noise at low Mach numbers [15]. The interest of Schwarzschild's technique is that it remains valid at any subsonic Mach number.

\subsection{Problem statement}

Schwarzschild's technique applied to turbulence-interaction noise has been thoroughly addressed by Amiet $[9,10,11]$. The principle of the procedure is summarized in Fig. 1, in terms of coordinates made non-dimensional by the half chord $c / 2$. The initial generic configuration involving a normal-velocity gust or upwash $w$ as incident disturbance (top plot) is split into half-plane problems of the type $\left(\mathcal{P}_{0}\right)$. The first one (bottom left) addresses the primary scattering of the incident gust by the leading edge and is solved on
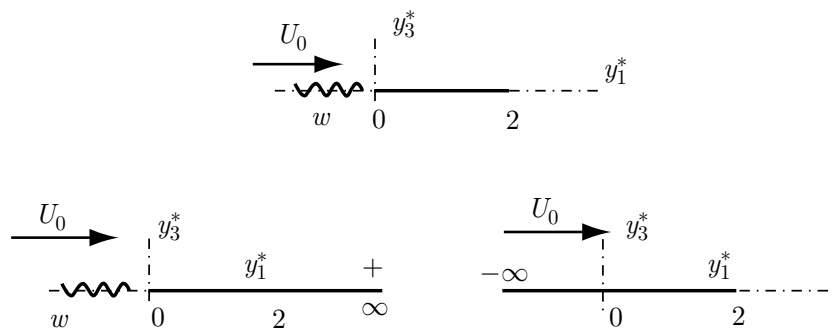

Figure 1: Turbulence-interaction noise model. Incident gust on a finite-chord airfoil (top), main scattering half-plane problem (bottom left) and trailing-edge correction (right). Coordinates made non-dimensional by the half chord, reference at the leading edge. 
(a)

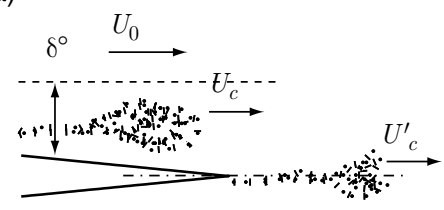

(c)

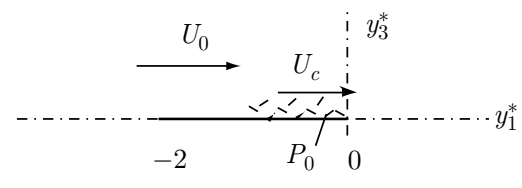

(b)

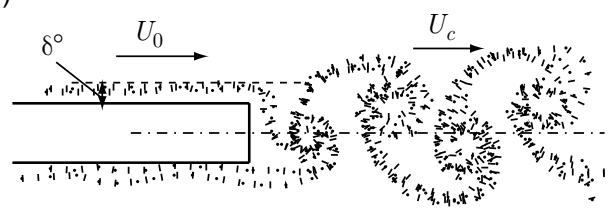

(d)

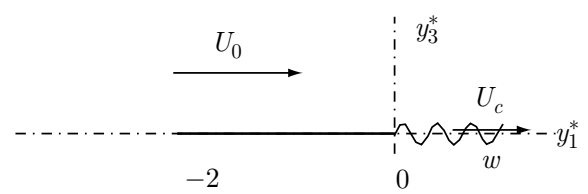

Figure 2: Trailing-edge vortex dynamics and model problems. (a): typical trailingedge flow for an attached turbulent boundary layer. (b): ideal vortexshedding pattern from a rectangular edge. (c) \& (d): equivalent generic configurations in non-dimensional variables, posed on the disturbance pressure and on the velocity, respectively.

the corresponding velocity potential. A first-order induced lift is derived from the relationship between the pressure and the potential. But the solution cannot satisfy the Kutta condition in the wake. This condition is better expressed on the pressure. It is enforced through a second-order correction defined such that the total pressure jump from the trailing edge and along the wake is exactly zero. This leads to a complementary problem of the form $\left(\mathcal{P}_{0}\right)$ (bottom right) written on the pressure at the trailing-edge, by means of a change of variables. These first two iterations have been shown to be enough in practice [11]. The solution holds a priori for frequencies such that $k c / \beta^{2}>0.4$ where $\beta^{2}=1-M_{0}^{2}$ and $M_{0}=U_{0} / c_{0}$ is the Mach number, for which typically the chord length starts to depart from acoustic compactness. Lower frequencies are not considered here.

Trailing-edge phenomena may appear more subtle in comparison because their dominant features strongly depend on the precise geometry of the trailing edge. In this paper two opposite situations are investigated. Pure trailing-edge noise refers to a configuration in which the incident turbulent scales and/or the boundary-layer thickness are large enough when compared to the physical thickness of the trailing edge. The latter is then considered as a sharp edge. The corresponding flow pattern is illustrated in Fig. 2-a, representative of most attached flows over well designed airfoils. This class of flows is addressed by the generic model of Fig. 2-c. The same strategy can be applied as for turbulence-interaction noise. The incident forcing on the trailing-edge is a wall-pressure gust of amplitude $P_{0}$ in the boundary layer of one side only, which in practice is the suction side of a loaded airfoil. The choice of the pressure is made again for the sake of imposing a Kutta condition. The latter can be understood in different ways, which remain a matter of controversy when resorting to simplified models expected to reproduce 
real-life flows. A full Kutta condition on the pressure jump is generally considered, even though this point remains a controversial issue $[4,16,17]$. The scattered pressure field around the edge involves contributions $\pm P_{0} / 2$ on each side of the plate, so that the pressure jump is continuous and zero. Another physically consistant choice is to assume that an incident vortical pattern in the boundary layer tends to follow its path in the wake with no noticeable modification. A Kutta condition of no cross-flow in the wake close to the edge is then only possible if an additional vorticity is shed in the wake which has the same effect as the image vortices of the incident pattern upstream of the edge. According to this interpretation, the pressure remains continuous and zero around the edge instead of the pressure jump, and the disturbance pressure $\pm P_{0}$ is distributed on each side of the airfoil. A factor 2 makes the difference between both assumptions, and the effect will be $6 d B$ more in the far-field noise with the second one, for the same incident turbulence. Apart from that, the procedure will be declined in the same way. The first Schwarzschild iteration is made assuming that the airfoil extends to infinity upstream. This is generally considered as sufficient [16] because boundary-layer flows involve smaller scales and typically higher frequencies than upstream turbulence impinging on a leading edge. Nevertheless a correction proposed as an extension in [12] is useful at low frequencies to include the effect of leading-edge back-scattering. This is achieved by deriving the potential from the disturbance pressure and by forcing the potential to zero in front of the leading edge (line $\left(z=y_{3}=0, x=y_{1}<-2\right)$ in Fig. 2-b) with a second-order correction which, again is solution of a Schwarzschild's problem. According to authors' experience with a set of airfoils tested at low speeds [18], the corrected solution together with the condition of zero pressure around the edge $(+6 \mathrm{~dB})$ produces the best agreement with measured data (see section 3); furthermore it ensures that Amiet's formulation coincides with asymptotic Howe's formulation at very high non-dimensional frequencies.

According to [13], the scattering process involved in vortex-shedding noise can be formalized as a reversed version of the mathematical problem for the turbulenceinteraction noise (Fig. 2-d). The best illustration would be to think of a time inversion or an inversion of the flow direction between both cases. In the case of vortex-shedding noise, vortical disturbances are shed from the trailing edge and evacuated downstream with their upwash continuation upstream forced to zero by the rigidity condition, whereas turbulence-interaction noise corresponds to an approaching vortical pattern the upwash of which is forced to zero for the same reasons. The additional feature of the reversed model is that the shed vorticity can be convected at a speed $U_{c}$ different from the free-stream velocity $U_{0}$. Furthermore, no back-scattering correction is needed because vortex shedding always occurs at high reduced frequencies for airfoil-like bodies. It must be noted that the Kutta condition makes no sense here, the pressure fluctuations induced by the vortex shedding being known as in phase opposition on both sides of the airfoil. In the model the same integrable singularity is accepted as for the turbulence-interaction noise. Strictly speaking the model assumptions are only reasonable if the formation of vortices occurs immediately in the near wake and is not displaced farther downstream. Therefore the model better addresses rectangular edge shapes. Its relevance in other geometrical designs might be questionable. 


\subsection{Closed-form far-field expressions}

The formulae of this section are written for the Power Spectral Density (PSD) of the acoustic pressure in the far field. They are derived in the reference papers for a stationary rectangular airfoil in a uniform flow and a reference taken at mid chord.

\subsubsection{Turbulence-interaction noise formulae}

The result for turbulence-interaction noise is first written as [9]

$$
\begin{aligned}
S_{p p}(\mathbf{x}, \omega)= & \left(\frac{k \rho_{0} c x_{3}}{2 S_{0}^{2}}\right)^{2} \\
& \times \pi U_{0} \frac{L}{2} \int_{-\infty}^{\infty}\left[\Phi_{w w}\left(\frac{\omega}{U_{0}}, k_{2}\right)\left|\mathcal{L}_{T I}\left(x_{1,} \frac{\omega}{U_{0}}, k_{2}\right)\right|^{2} \frac{\sin ^{2}\left[\left(\frac{k x_{2}}{S_{0}}-k_{2}\right) \frac{L}{2}\right]}{\pi \frac{L}{2}\left(\frac{k x_{2}}{S_{0}}-k_{2}\right)^{2}}\right] d k_{2}
\end{aligned}
$$

where $\mathcal{L}_{T I}$ is the non-dimensional chordwise aeroacoustic transfer function

$$
\mathcal{L}_{T I}\left(x_{1}, \frac{\omega}{U_{0}}, k_{2}\right)=\int_{-1}^{1} \frac{\tilde{\ell}^{A} e^{-i k_{2}^{*} y_{2}^{*}}}{2 \pi \rho_{0} w_{0}} e^{-i \bar{\mu}\left(\frac{x_{1}}{S_{0}}-M\right) y_{1}^{*}} d y_{1}^{*}
$$

and $\widetilde{\ell}^{A}$ is the unsteady lift induced by the gust of amplitude $w_{0}$, as determined by Schwarzschild's procedure. $S_{0}=\left[x_{1}^{2}+\beta^{2}\left(x_{2}^{2}+x_{3}^{2}\right)\right]^{1 / 2}$ is a convection-corrected distance, close to the geometrical distance $R$ if $M_{0}$ is small. $L$ is the span, $k=\omega / c_{0}$ is the acoustic wavenumber, and $\rho_{0}$ the air density. $\Phi_{w w}\left(k_{1}, k_{2}\right)$ is the two-dimensional wavenumber spectrum of the incident turbulence, and $k_{1}=\omega / U_{0}$ is imposed by the frozen-turbulence assumption. The function $\mathcal{L}$ has different expressions for the subcritical and supercritical gusts corresponding to subsonic or supersonic phase speeds of their trace on the plate with respect to the incident mean flow, respectively. Very often only the large aspect-ratio approximation of eq. (1) is considered:

$$
S_{p p}(\mathbf{x}, \omega)=\left(\frac{\rho_{0} k c x_{3}}{2 S_{0}^{2}}\right)^{2} \pi U_{0} \frac{L}{2} \Phi_{w w}\left(\frac{\omega}{U_{0}}, \frac{k x_{2}}{S_{0}}\right) \mid \mathcal{L}_{T I}\left(x_{1}, \frac{\omega}{U_{0}}, \frac{k x_{2}}{S_{0}}\right)^{2}
$$

and the function $\mathcal{L}$ needs to be specified only for the parallel gust $k_{2}=0$ and then to be corrected by Graham's similarity rules. In that case each involved oblique gust is always supercritical and radiates at a well-defined inclination angle from the surface. The contribution of the sub-critical gusts is only non-zero for a finite span, which is the case 
in practice. Formulae for the distributed lift have been provided for instance by Mish \& Devenport [19]. A complete set of closed-form expressions including $\mathcal{L}$ in both cases of sub- and supercritical gusts is reproduced here from [20]. This transfer function is found to be the sum of two contributions $\mathcal{L}=\mathcal{L}_{1}+\mathcal{L}_{2}$. For sub-critical gusts corresponding to a value of Graham's parameter $\Theta_{0}=k_{1}^{*} M_{0} /\left(\beta k_{2}^{*}\right)$ smaller than 1:

$$
\begin{gathered}
\mathcal{L}_{1}=-\frac{1}{\pi} \sqrt{\frac{2}{\left(k_{1}^{*}+i \beta^{2} \bar{\kappa}^{\prime}\right) i \Theta_{3}} e^{-i \Theta_{2}} E\left[2 i \Theta_{3}\right],} \\
\mathcal{L}_{2}=\frac{e^{-i \Theta_{2}}}{\pi \sqrt{2 \pi\left(k_{1}^{*}+i \beta^{2} \bar{\kappa}^{\prime}\right)} \Theta_{3}} \\
\quad \times\left\{1-e^{-2 \Theta_{3}}-\operatorname{erf}\left(\sqrt{4 \bar{\kappa}^{\prime}}\right)+2 e^{-2 \Theta_{3}} \sqrt{\frac{\bar{\kappa}^{\prime}}{i \bar{\kappa}^{\prime}+\bar{\mu} x_{1} / S_{0}}} E\left[2\left(i \bar{\kappa}^{\prime}+\bar{\mu} x_{1} / S_{0}\right)\right]\right\},
\end{gathered}
$$

where $\Theta_{2}=\bar{\mu}\left(M_{0}-x_{1} S_{0}\right)-\frac{\pi}{4}$

$$
\begin{gathered}
\Theta_{3}=\bar{\kappa}^{\prime}+i \bar{\mu} \frac{x_{1}}{S_{0}}, \quad \bar{\kappa}^{\prime}=\sqrt{\frac{k_{2}^{* 2}}{\beta^{2}}-\bar{\mu}^{2}}, \quad \bar{\kappa}^{\prime 2}=\bar{\mu}^{2}\left(\frac{1}{\Theta_{0}^{2}}-1\right), \\
\bar{\mu}=\frac{k c}{2 \beta^{2}}=\frac{k_{1}^{*} M_{0}}{\beta^{2}}, \quad k_{j}^{*}=\frac{k_{j} c}{2} .
\end{gathered}
$$

E is related to Fresnel integrals: $E(\xi)=\int_{0}^{\xi} \frac{e^{i t}}{\sqrt{2 \pi t}} d t$

The alternative expressions for a supercritical oblique $\left(\Theta_{0}>1\right.$ and $\left.\bar{\kappa}^{\prime 2}<0\right)$ are deduced from the preceding ones by simply changing $i \bar{\kappa}^{\prime}$ in $\bar{\kappa}$ and by replacing the error function erf $(\sqrt{.})$ by the function $(1-i) E$ :

$$
\begin{gathered}
\mathcal{L}_{1}=-\frac{1}{\pi} \sqrt{\frac{2}{\left(k_{1}^{*}+\beta^{2} \bar{\kappa}\right) \Theta_{4}}} e^{-i \Theta_{2}} E\left[2 \Theta_{4}\right], \\
\mathcal{L}_{2}=\frac{e^{-i \Theta_{2}}}{\pi \Theta_{4} \sqrt{2 \pi\left(k_{1}^{*}+\beta^{2} \bar{\kappa}\right)}} \\
\quad \times\left\{i\left(1-e^{2 i \Theta_{4}}\right)-(1+i)\left[E(4 \bar{\kappa})-e^{2 i \Theta_{4}} \sqrt{\frac{2 \bar{\kappa}}{\bar{\kappa}+\bar{\mu} x_{1} / S_{0}}} E\left[2\left(\bar{\kappa}+\bar{\mu} x_{1} / S_{0}\right)\right]\right]\right\}
\end{gathered}
$$


with

$$
\Theta_{4}=\bar{\kappa}-\bar{\mu} \frac{x_{1}}{S_{0}}
$$

\subsubsection{Trailing-edge noise formulae}

The two-step Schwarzschild's solution for trailing-edge noise is taken here from reference [12], extending Amiet's approach. It must be noted that alternative formulations, first reviewed by Howe [4], have been proposed in the literature [3], and that a low-Mach number formulation including the effect of finite chord is found in [15]. Amiet's approach is chosen because it involves the same basic assumptions as for turbulence-interaction noise. The sound pressure PSD reads

$$
S_{p p}(\mathbf{x}, \omega)=\left(\frac{k c x_{3}}{4 \pi S_{0}^{2}}\right)^{2} 2 c \int_{-\infty}^{\infty} \Pi_{0}\left(\frac{\omega}{U_{c}}, k_{2}\right) \frac{\sin ^{2}\left[\frac{L}{c}\left(k_{2}^{*}-\bar{k} \frac{x_{2}}{S_{0}}\right)\right]}{\left(k_{2}^{*}-\bar{k} \frac{x_{2}}{S_{0}}\right)^{2}} \mid \mathcal{I}\left(\frac{\bar{\omega}}{U_{c}},\left.k_{2}^{*}\right|^{2} d k_{2}^{*}\right.
$$

with $\bar{k}=k c / 2$. The corresponding large aspect-ratio approximation is

$$
S_{p p}(\mathbf{x}, \omega)=\left(\frac{k c x_{3}}{4 \pi S_{0}^{2}}\right)^{2} 2 \pi L \mid \mathcal{I}\left(\frac{\bar{\omega}}{U_{c}},\left.\bar{\kappa} \frac{x_{2}}{S_{0}}\right|^{2} \prod_{0}\left(\frac{\omega}{U_{c}}, k \frac{x_{2}}{S_{0}}\right)\right.
$$

In eqs. (7) and (8) the statistical function $\Pi_{0}$ is related to the wall-pressure spectrum $\Phi_{p p}$ closely upstream of the trailing-edge for assumed homogeneous boundary-layer turbulence and the associated spanwise correlation length $l_{y}$ by

$$
\prod_{0}\left(\frac{\omega}{U_{c}}, k_{2}\right)=\frac{1}{\pi} \Phi_{p p}(\omega) l_{y}\left(k_{2}, \omega\right) \quad l_{y}\left(k_{2}, \omega\right)=\int_{0}^{\infty} \sqrt{\gamma^{2}\left(\eta_{2}, \omega\right)} \cos \left(k_{2} \eta_{2}\right) d \eta_{2}
$$

$\gamma^{2}$ being the spanwise coherence between points separated by a distance $\eta_{2}$. Formulae for the aeroacoustic transfer function $\mathcal{I}=\mathcal{I}_{1}+\mathcal{I}_{2}$ are listed below. The first-order term is obtained as

$$
\mathcal{I}_{1}=-\frac{e^{2 i C}}{i C}\left\{(1+i) e^{-2 i C} \sqrt{\frac{B}{B-C}} E^{*}[2(B-C)]-(1+i) E^{*}[2 B]+1\right\}
$$

with

$$
C=\bar{K}_{1}-\bar{\mu}\left(\frac{x_{1}}{S_{0}}-M_{0}\right) \quad B=\bar{K}_{1}+M_{0} \bar{\mu}+\bar{\kappa} \quad \bar{\kappa}^{2}=\bar{\mu}^{2}-\frac{k_{2}^{*_{2}}}{\beta^{2}} \quad \bar{K}_{1}=\frac{\omega c}{2 U_{c}}=K_{1} \cdot \frac{c}{2}
$$

and is identical to Amiet's in the special case $k_{2}^{*}=0$. 
The leading-edge back-scattering correction is

$$
\frac{1}{H} \mathcal{I}_{2}=\left\{e^{4 i \bar{\kappa}}\left[1-(1+i) E^{*}(4 \bar{\kappa})\right]\right\}^{c}-e^{2 i D}+i\left[D+k_{1}^{*}+M_{0} \bar{\mu}-\bar{\kappa}\right] G
$$

with

$$
\begin{aligned}
H= & \frac{(1+i) e^{-4 i \bar{\kappa}}\left(1-\Theta^{2}\right)}{2 \sqrt{\pi}(\alpha-1) k_{1}^{*} \sqrt{B}} \quad D=\bar{\kappa}-\bar{\mu} x_{1} / S_{0} \quad \Theta=\sqrt{\frac{\overline{K_{1}}+\bar{\mu} M_{0}+\bar{\kappa}}{k_{1}^{*}+\bar{\mu} M_{0}+\bar{\kappa}}} \quad \alpha=\frac{U_{0}}{U_{c}} \\
G= & (1+\varepsilon) e^{i(2 \bar{\kappa}+D)} \frac{\sin (D-2 \bar{\kappa})}{D-2 \bar{\kappa}}+(1-\varepsilon) e^{i(-2 \bar{\kappa}+D)} \frac{\sin (D+2 \bar{\kappa})}{D+2 \bar{\kappa}} \\
& +\frac{(1+\varepsilon)(1-i)}{2(D-2 \bar{\kappa})} e^{4 i \bar{\kappa}} E^{*}(4 \bar{\kappa})-\frac{(1-\varepsilon)(1+i)}{2(D+2 \bar{\kappa})} e^{-4 i \bar{\kappa}} E(4 \bar{\kappa}) \\
& +\frac{e^{2 i D}}{2} \sqrt{\frac{2 \bar{\kappa}}{D}} E^{*}(2 D)\left[\frac{(1+i)(1-\varepsilon)}{D+2 \bar{\kappa}}-\frac{(1-i)(1+\varepsilon)}{D-2 \bar{\kappa}}\right]
\end{aligned}
$$

Here the notation $\{\cdot\}^{c}$ means that the imaginary part must be multiplied by the factor $\varepsilon=(\sqrt{1+1 /(4 \bar{\mu})})^{-1}$.

For the sub-critical gusts the solutions read

$$
\begin{aligned}
\mathcal{I}_{1}= & -\frac{e^{-2 i C}}{i C} \\
& \left\{e^{-2 i C} \sqrt{\frac{A^{\prime}{ }_{1}}{\bar{\mu}\left(x_{1} / S_{0}\right)-i \bar{\kappa}^{\prime}}} \Phi^{\circ}\left(\left[2 i\left(\bar{\mu}\left(x_{1} / S_{0}\right)-i \bar{\kappa}^{\prime}\right)\right]^{1 / 2}\right)-\Phi^{\circ}\left(\left[2 i A_{1}^{\prime}\right]^{1 / 2}\right)+1\right\}
\end{aligned}
$$

and

$$
\begin{aligned}
\mathcal{I}_{2} & =\frac{e^{-2 i B^{\prime}}}{B^{\prime}} H^{\prime} \\
& \left\{A^{\prime}\left(e^{2 i B^{\prime}}\left[1-\operatorname{erf}\left(\sqrt{4 \bar{\kappa}^{\prime}}\right)\right]-1\right)+\sqrt{2 \bar{\kappa}^{\prime}}\left(k_{1}^{*}+\left(M_{0}-\frac{x_{1}}{S_{0}}\right) \bar{\mu}\right) \frac{\Phi^{\circ}\left(\sqrt{-2 i B^{\prime}}\right)}{\sqrt{-i B^{\prime}}}\right\}
\end{aligned}
$$

where $\Phi^{\circ}$ stands for the complementary error function of complex argument and where

$$
\begin{aligned}
H^{\prime}=\frac{(1+i)\left(1-\Theta^{\prime 2}\right)}{2 \sqrt{\pi}(\alpha-1) k_{1}^{*} \sqrt{A_{1}^{\prime}}} & A_{1}^{\prime}=\bar{K}_{1}+M_{0} \bar{\mu}-i \bar{\kappa}^{\prime} \\
A^{\prime}=k_{1}^{*}+M_{0} \bar{\mu}-i \bar{\kappa}^{\prime} & \Theta^{\prime}=\sqrt{\frac{A_{1}^{\prime}}{A^{\prime}}} \\
B^{\prime}=\bar{\mu}\left(\frac{x_{1}}{S_{0}}\right)-i \bar{\kappa}^{\prime} &
\end{aligned}
$$


Very often the leading-edge back-scattering correction (terms of index 2) is not needed by virtue of the high frequencies encountered in usual trailing-edge noise applications. However lower frequencies are produced by nearly separated flows over highly loaded airfoils, for which the model remains reliable, and the formulae for the correction are given here for completeness.

\subsubsection{Vortex-shedding noise formulae}

The first-order acoustic pressure PSD for vortex-shedding noise has been derived in [13] according to the large-aspect ratio approximation in the special case of the parallel gust and of an observer in the mid-span plane, for the sake of simplicity. Indeed the two involved velocities $U_{0}$ and $U_{c}$ make the formulation more complicated. Furthermore vortexshedding is known to be correlated over a significant spanwise extent, typically 6 or 7 times the edge thickness, and makes the parallel gust dominant. The expression reads

$$
S_{p p}(\mathbf{x}, \omega)=\left(\frac{\rho_{0} k c x_{3} U_{0}}{2 S_{0}^{2}}\right)^{2} \frac{L}{2} S_{w w}(\omega) l_{y}(\omega)\left|\mathcal{L}_{V K}\right|^{2}
$$

where the normalized radiation integral at angular frequency $\omega$ is given by

$$
\begin{aligned}
\mathcal{L}_{V K}= & -\frac{(1+i)}{\pi K_{1}^{*}} \\
& \left\{\sqrt{\frac{\Theta_{1}}{\Theta_{1}-\Theta_{2}^{\prime}}} E^{*}\left[2\left(\Theta_{1}-\Theta_{2}^{\prime}\right)\right]\left(1-\frac{\bar{K}_{1}-k_{1}^{*}}{\Theta_{2}^{\prime}}\right)+\left(\bar{K}_{1}-k_{1}^{*}\right) \frac{e^{2 i \Theta_{2}^{\prime}}}{\Theta_{2}^{\prime}} E^{*}\left[2 \Theta_{1}\right]\right\}
\end{aligned}
$$

with

$$
\Theta_{2}^{\prime}=\bar{K}_{1}-\bar{\mu}\left(\frac{x_{1}}{S_{0}}-M_{0}\right) \text { and } \Theta_{1}=\bar{K}_{1}+\bar{\mu}\left(1+M_{0}\right)
$$

Here $S_{w w}$ is the PSD of the upwash velocity $w, l_{y}$ the corresponding spanwise correlation length and $K_{1}^{*}=\bar{K}_{1}\left[1-M_{0}^{2}\left(1-k_{1} / K_{1}\right)^{2}\right]^{1 / 2}{ }^{y}$ (note that again the bar or the asterisk on a wavenumber corresponds to a multiplication by $c / 2$ ). It can be noted that eq. (14) reduces to the main term of Amiet's result for turbulence-interaction noise in the special case $\bar{K}_{1}=K_{1}^{*}=k_{1}^{*}$, provided that the flow is reversed, thus changing the sign of the Mach number.

\subsubsection{Scaling laws}

Aeroacoustic phenomena are expected to exhibit higher frequencies and levels with increasing mean-flow speeds. Therefore similarity or scaling laws are sought by introducing some non-dimensional frequency or Strouhal number and dividing the PSD of the acoustic pressure by $U_{0}^{n}$ where the exponent $n$ is to be determined. Such a postprocessing is guided by theoretical arguments. Indeed the common feature of all 
analytical models is that the acoustic intensity is proportional to the mean square value of the forcing disturbance, $S_{w w}$ or $\Phi_{p p}$, to its spanwise correlation length $l_{y}$ and to the spanwise extent of the edge $L$. Furthermore the incident disturbances must have the properties of homogeneous and stationary random processes. A dimensionless form of the formulae is better obtained in the mid-span plane and from the large aspect-ratio approximations of the results assuming that the ratio $l_{y} / L$ is small, as

$$
\frac{2 S_{0}^{2} S_{p p}(\mathbf{x}, \omega)}{L l_{y}\left(\rho_{0} U_{0}\right)^{2} S_{w w}}=\left(\frac{k c x_{3}}{2 S_{0}}\right)^{2}|\mathcal{L}|^{2} \quad \frac{2 \pi^{2} S_{0}^{2} S_{p p}(\mathbf{x}, \omega)}{L l_{y} \Phi_{p p}}=\left(\frac{k c x_{3}}{2 S_{0}}\right)^{2}|\mathcal{I}|^{2}
$$

where $\mathcal{L}$ now stands for the response function of either turbulence-interaction noise or vortex-shedding noise. These expressions can be used to scale and compare the experimental results gathered from different setups by different authors, as shown in the next section. The analytical solutions for the response functions $\mathcal{L}$ or $\mathcal{I}$ lead to simple asymptotic trends in both limits of low and high frequencies, which for subsonic Mach numbers are expressed by the Helmholtz number $k c$. Though the vanishing frequencies are not compatible with the high-frequency assumption inherent to Schwarzschild's technique, it can be guessed that $\mathcal{L}$ is continued to a constant of order 1 . In the limit of high frequencies, $|\mathcal{L}|^{2}$ becomes proportional to $(k c)^{-2} M_{0}$.

\section{EXPERIMENTAL VALIDATION OF THE BROADBAND-NOISE MODELS}

An experimental configuration both simple enough and representative of subsonic fan blades must be selected for the validation of the analytical models described in the previous section. Each mechanism should be investigated in the same controlled flow conditions, by adjusting the angle of attack or by changing the airfoil shape. As mentioned by Moreau et al. [21], such requirements are best achieved by placing an instrumented airfoil mock-up in a large quiet environment such as the open free-jet anechoic wind tunnels of Fig. 3, in order to keep extra noise sources as low as possible to avoid covering the trailing edge noise over a significant frequency range. The same protocol has been used by previous investigators, for instance Paterson \& Amiet [10] or Brooks \& Hodgson [22]. The air-supply device must provide stable and clean airflow with a nozzle setup that makes the known inlet velocity profile as flat as possible. It must be quiet enough and free of vibrations in the test section. The airfoil is held between two horizontal side-plates fixed to the nozzle of the wind tunnel. The mock-up aspect ratio (span over chord length) is at least 3 in order to minimize the three-dimensional effects [18] and the contamination by spurious sources at the junctions when dealing with mid-span measurements. The plate edges may need to be designed to reduce their trailing-edge noise, and the diffraction by the nozzle corners resulting in a distortion of the airfoil noise directivity may be corrected [23].

Apart from the background noise generated by the flow on the side-plates and at the junctions between the tested airfoil and the side-plates, the use of rectangular-airfoil 

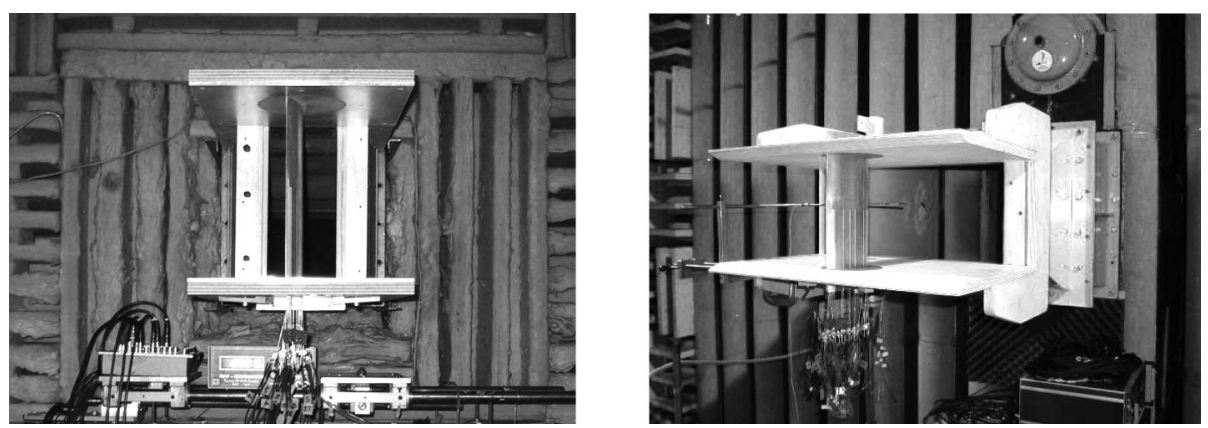

Figure 3: Experimental setups of ECL in open-jet anechoic wind tunnels with narrow (WT1) and large (WT2) rectangular nozzles, featuring the instrumented airfoils. Remote-microphone probes for wall-pressure measurements, coupling a capillary tube inside the mock-up and an external microphone, are visible below the lower side-plates.

setups also involves a mean flow deviation due to the lateral momentum injection at non-zero angle of attack. Therefore the effective flow at a given geometrical angle of attack is different from the flow at the same angle in an infinite stream. This must be accounted for if the results are compared to simulations of the flow, either by including the nozzle in the CFD mesh to reproduce the deviation, or, if possible, by adjusting the angle of attack to get the best fit between measured and predicted mean-pressure coeffi cient distributions over the airfoil. Typically in the narrow wind tunnel WT1, the deviation effect is equivalent to a cascade effect and the flow cannot be compared to a configuration in infinite stream, even by an adjustment of the angle of attack.

\subsection{Turbulence-interaction noise}

For turbulence-interaction noise investigation, a grid is placed in the nozzle upstream of the test section. Such a grid has a known square mesh and grid thickness which ensure a homogeneous isotropic turbulence at some distance, with known turbulence intensity $\bar{w}^{2}$ and energy integral scale $\Lambda$. These parameters determine the needed velocity spectrum $\Phi_{w w}$ according to Liepmann or von Kármán models. Based on the assumption of ideal incident turbulence, asymptotic scaling laws can be explicitly known. For an assessment of the models in the mid-span plane the upwash velocity spectrum $S_{w w}$ and the spanwise correlation length are related to $\Phi_{w w}$ by $\Phi_{w w}=U_{0} S_{w w} l_{y} / \pi$ and are described by functions $\mathcal{F}$ and $\mathcal{G}$ such that

$$
S_{w w}=\frac{\bar{w}^{2}}{U_{0}} \frac{\Lambda}{2 \pi} \mathcal{F}\left(k_{1} \Lambda\right) \quad l_{y}=a \Lambda \mathcal{G}\left(k_{1} \Lambda\right)
$$

where $a$ is a constant. The function $\mathcal{F}$ goes to 1 at vanishing frequencies and asymptotically decreases as $\left(k_{1} \Lambda\right)^{-n}$ at high frequencies, with $n=2$ for the Liepmann 
spectrum (and $n=5 / 3$ for the von Kármán spectrum). In the same way $\mathcal{G}$ increases as $\left(k_{1} \Lambda\right)^{2}$ at very low frequencies and decreases asymptotically as $\left(k_{1} \Lambda\right)^{-1}$ at very high frequencies. The low-frequency and high-frequency asymptotic trends in homogeneous and isotropic turbulence are thus expected to obey the general laws, respectively

$$
\frac{c_{0} S_{0}^{2} S_{p p}}{\tau^{2} \rho_{0}^{2} U_{0}^{4} L c^{2}} \propto\left(\frac{x_{3}}{S_{0}}\right)^{2} \frac{U_{0}}{c_{0}}\left(k_{1} \Lambda\right)^{4} \quad \frac{c_{0} S_{0}^{2} S_{p p}}{\tau^{2} \rho_{0}^{2} U_{0}^{4} L c^{2}} \propto\left(\frac{x_{3}}{S_{0}}\right)^{2}\left(\frac{\Lambda}{c}\right)^{2} \frac{1}{\left(k_{1} \Lambda\right)^{3}}
$$

This is only an indicative result since in practice the asymptotic regimes may be obtained for different frequencies in the incident turbulence and in the aeroacoustic response.

Typical results measured at ECL at three different speeds with isolated airfoils of different shapes are first reported in Fig. 4 (top plots). The airfoils are a NACA-0012 of $c=10 \mathrm{~cm}$ chord length, a flat plate of same chord and of relative thickness $h / c=3 \%$, and a slightly cambered Controlled-Diffusion (CD) airfoil (maximum thickness around mid-chord $h / c \simeq 4 \%, c=13 \mathrm{~cm}$, camber $24^{\circ}$ ). The acoustic PSD is normalized by the quantity $U_{0}^{4}$ or $5 L c^{2} / R^{2}$ justified by the asymptotic scaling laws, $R$ being the geometrical distance (note that $S_{0}$ is close to $R$ ). The turbulence is generated by the same grid put upstream of the nozzle, ensuring a turbulence rate $\tau$ of $5 \%$ and an integral length scale $\Lambda$ of $0.9 \mathrm{~cm}$. The thin bodies produce equivalent noise levels and are found intrinsically louder than the NACA-0012 at high frequencies, whereas all results coincide at low frequencies. For the same body, the $n=5$ scaling produces a slightly better collapse at low frequencies, and the $n=4$ scaling is much better at high frequencies. This corresponds to integrated energy levels scaling with the powers 6 and 5 of the incoming flow speed, respectively. The change is a known effect of non compactness in the chordwise direction. The results for the NACA-0012 are also compared to previously reported measurements by Paterson \& Amiet [10] with a larger chord and higher flow speeds ranging from 40 to $190 \mathrm{~m} / \mathrm{s}$ in Fig. 4-(c, d). Since the turbulence grid is not the same in both experiments, the frequency range has been re-scaled by including the ratio $\xi$ of the integral length scales and the acoustic PSD is now also normalized differently according to eqs. (15). Despite the different experimental conditions, all normalized data collapse fairly well, especially with the $U_{0}^{4}$-scaling of the PSD.

The theoretical slope $\left(k_{1} \Lambda\right)^{-3}$ of eq. (15) is also plotted as the dashed line in Fig. 4-b $\& \mathrm{~d}$. It is found in the measurements for thin airfoils, and only at the beginning of the corresponding high-frequency range with the NACA-0012, for which the data depart from the trend at higher frequencies. This is attributed to a thickness effect recognized as responsible for a more rapid drop and already reported by previous investigators, typically dealing with thick wind-turbine blades [24, 25].

The measurements with the three aforementioned airfoil shapes are compared to analytical predictions from eq. (1) in Fig. 5. A good agreement is found with the thin bodies but the noise from the NACA-0012 is overestimated by an amount of up to $10 d B$ at the highest frequencies due to the thickness effect. Additional tests made on 
(a)

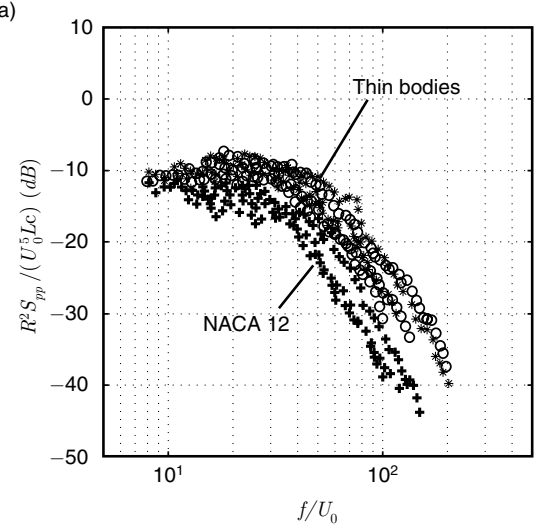

(c)

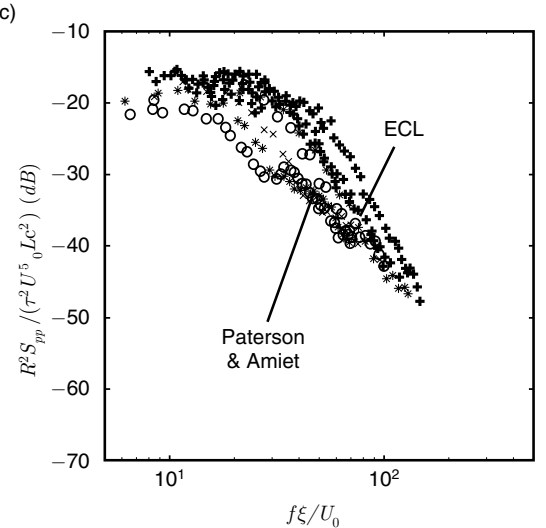

(b)

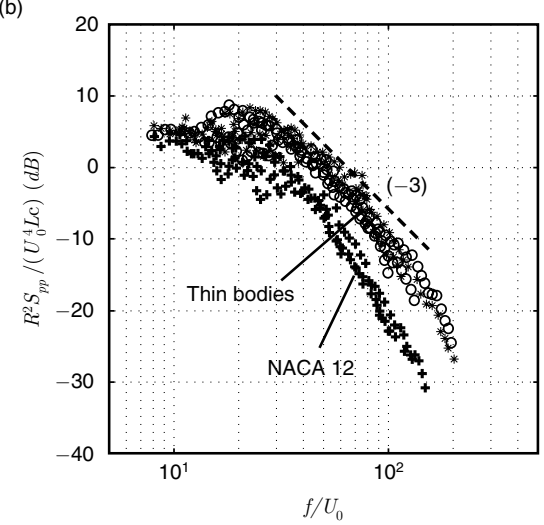

(d)

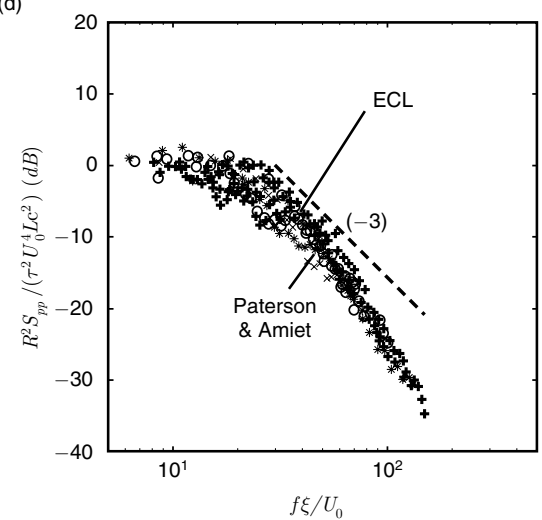

Figure 4: $\quad$ (a) \& (b): experimental data from ECL setups with a NACA-12 airfoil (+) and thin airfoils or plates (other symbols). Flow speeds 20,30 and $40 \mathrm{~m} / \mathrm{s}$. Normalized data based on $\mathrm{U}_{0}^{5}$ and $\mathrm{U}_{0}^{4}$ scaling laws. (c) \& (d): same data re-normalized for the NACA 12 airfoil only (+), compared to Paterson \& Amiet's results (other symbols) at different flow speeds [10] (grid-tuned frequency axis).

the tunnel WT1 with another thin and slightly cambered 'optimized' airfoil (chord length $13 \mathrm{~cm}$, relative thickness less than 3\%) are reported in Fig. 5-b for the three flow speeds 20, 30 and $40 \mathrm{~m} / \mathrm{s}$, over an extended frequency range. Again the agreement is very good, and the model nicely reproduces the dips and humps in the spectral shape, which are a known effect of non-compactness. At the low Mach numbers considered here, for which $\beta^{2} \simeq 1$, they arise at the same reduced frequencies $k c=2 \pi$ and $k c=4 \pi$ whatever the flow speed might be.

It must be noted that the overprediction for the NACA-0012 is only noticed in Paterson $\&$ Amiet's results at the lowest speeds, whereas the agreement (not shown here) is very 
(a)

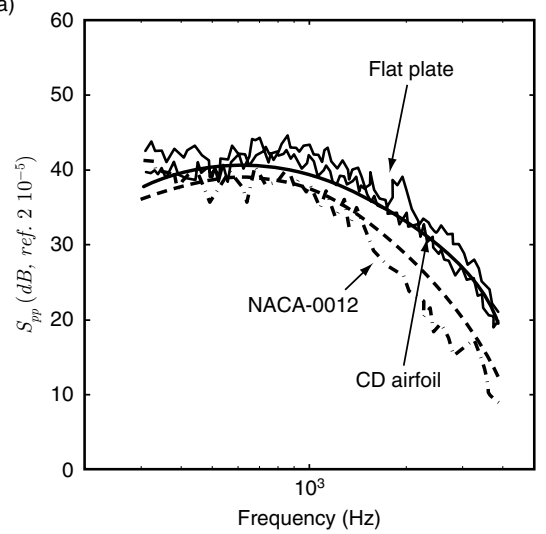

(b)

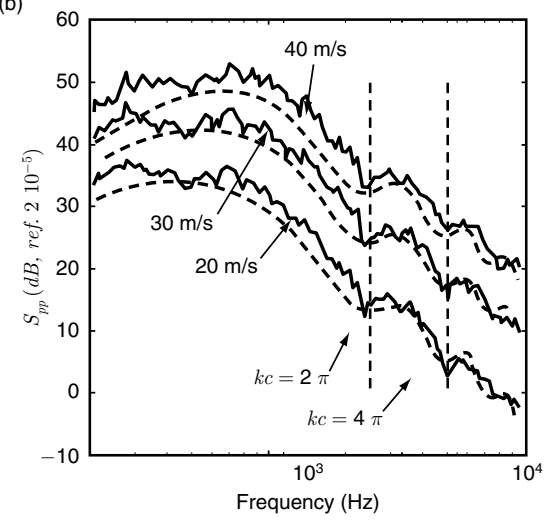

Figure 5: Predicted versus measured turbulence-interaction noise spectra. Dimensional data from ECL wind tunnel WT1. (a): flat plate, CD airfoil and NACA-0012 airfoil, flow speed $30 \mathrm{~m} / \mathrm{s}$, thin-airfoil theory as the thick line. Rapid-distortion theory correction according to [26] in dashed line. (b): measurements (cont.) versus predictions (dashed) for a thin optimized airfoil, flow speeds 20,30 and $40 \mathrm{~m} / \mathrm{s}$, featuring the non-compactness dips and humps.

good over the whole investigated frequency range at the highest speeds [10]. In fact the integral length scale of the incoming turbulence in a setup is nearly independent of flow speed, and high frequencies are excited by statistically larger eddies in the turbulence as the mean flow speed increases. The thickness effect is expected for small eddies, intuitively of the same order of and smaller than the leading-edge thickness of the airfoil, or the leadingedge curvature radius. Thus the occurence of noise reduction due to thickness effect is delayed at higher frequencies as the flow accelerates. The net result is that the turbulenceinteraction noise model fails at low speeds and high frequencies for thick airfoils. The amount of reduction at high frequencies should be different on different installations. But the ratio of thickness to eddy size is precisely nearly the same (around 1) in both experiments of Fig. 4-(c, d), leading to a good collapse. For a deeper investigation, an assessment of the reduction due to thickness effect measured by different authors is reported in Fig. 6, from references [10, 24, 26, 27]. The difference between Amiet's theory for thin airfoils and the measurements, or the difference between the sound radiation from airfoils of various relative thicknesses in the same flow, is analyzed as a far-field response reduction due to thickness effect. The reduction is divided by the ratio $(e / c) /(e / c)_{r e f}$ and plotted as a function of the variable $f \zeta / U_{0}$ where $\zeta=(\Lambda / c)_{r e f} /(\Lambda / c)$, the index 'ref' standing for the NACA-0012 airfoil in Paterson \& Amiet's experiment taken as reference. In Olsen's experiment [27], the rectangular airfoil is placed in the free turbulent jet from a circular nozzle, at a downstream distance of four diameters. In this conditions the value of $\Lambda$ has been taken as $20 \%$ of the diameter and the equivalent incoming flow speed taken as the local mean convection speed, $60 \%$ of the jet speed at the nozzle exit. The data of 


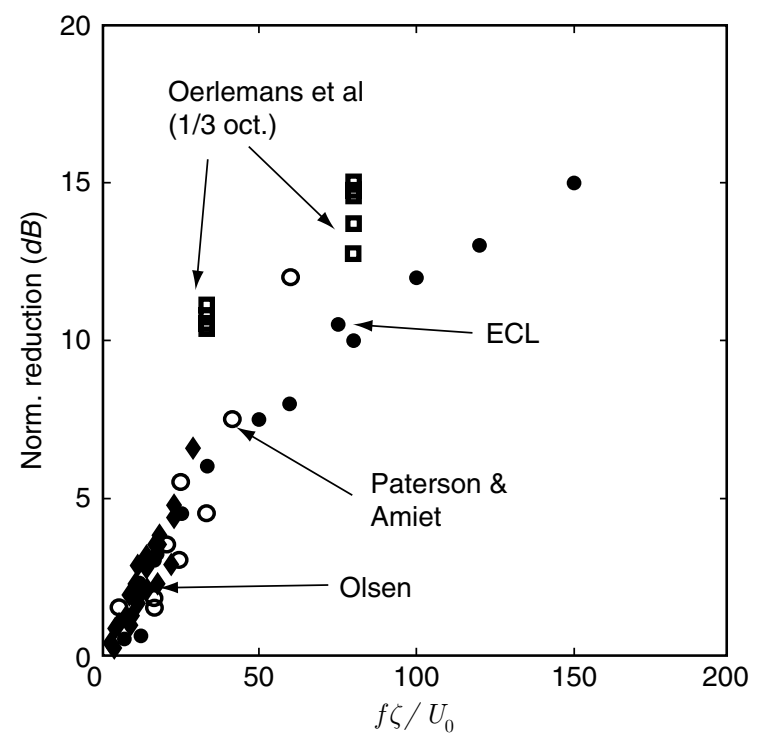

Figure 6: Amount of far-field response reduction of an airfoil to incident turbulence as a function of leading-edge thickness and frequency. Normalized data. NACA-0012 airfoil according to Paterson \& Amiet [10] and ECL results, wind-turbine airfoils from Oerlemans \& Migliore [24] and Olsen's results for airfoils in a turbulent round jet [27].

Oerlemans \& Migliore [24] are mentioned for completeness but may be not comparable because all parameters are not available for the scaling. The other data are in a reasonable linear collapse, except for large values of $f \zeta / U_{0}$. The result is again quite remarkable in view of the very different flow conditions. It suggests that the noise reduction $\Delta_{d B}$ due to thickness effect is proportional to the relative thickness and inversely proportional to the ratio $\Lambda / e$ and the flow speed, with the assumption of flow similarity:

$$
\Delta_{d B} \propto \frac{(e / c)}{(e / c)_{r e f}} \frac{f}{U_{0}} \frac{(\Lambda / e)_{r e f}}{(\Lambda / e)}
$$

over the range of collapse.

In order to cope with the limitation of thin-airfoil assumption, thickness corrections have been proposed in the literature $[26,28]$. The effect of an indicative correction of the incident turbulence spectrum based on Hunt's rapid distortion theory (RDT) at the leading-edge of the NACA-0012, proposed by Moreau et al [26] is superimposed on the results of Fig. 5-a. The agreement with the measurement is significantly improved, suggesting that quite simple extensions of original Amiet's formulation are possible. 


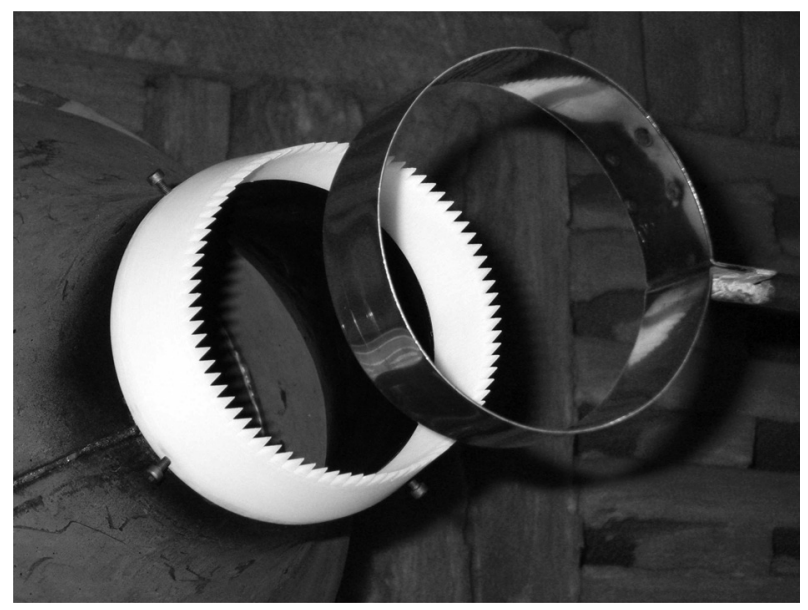

Figure 7: The jet-ring arrangement as an alternative experimental setup for studying turbulence-interaction noise from an airfoil in circular geometry, from [20]. The serrations on the nozzle suppress the acoustic back-reaction from the ring, which would induce self-sustained oscillations involving the jet instability modes.

\subsubsection{Validation off the mid-span plane}

The experimental setups of Fig. 3 allow measuring the sound only in the mid-span plane for which the major contribution arises from the turbulent gusts with wavefronts parallel to the leading edge ( $k_{2}=0$ in the general expressions). Oblique radiation o the mid-span plane enforced by oblique incident gusts is more difficult to assess because of the installation constraints. A recent experiment in circular geometry with a flat ring placed in the mixing layer of a turbulent jet with the same diameter has partly overcome this restriction [20]. Amiet's formulation was extended in circular geometry, arguing that at high frequencies and for small disturbances the unsteady lift response of the ring to helical gusts is the same as the response of a rectangular airfoil to plane gusts. In such a configuration the far-field microphone is measuring sounds radiated at oblique directions from the curved surface of the ring. The final expression for the PSD of the acoustic pressure in the far field reads

$$
S_{p p}(\mathbf{x}, \omega)=\left(\frac{\pi k c \rho_{0}}{2 S_{0}^{2}}\right)^{2} 2 \pi r_{0} U \sum_{n=-\infty}^{\infty} \Phi_{w w}\left(\frac{\omega}{U}, \kappa_{n}\right)\left|\mathcal{L}_{T I}\left(x_{1}, \frac{\omega}{U}, \kappa_{n}\right)\right|^{2} T^{2}\left[J_{n}^{\prime}\left(\frac{k r_{0} T}{S_{0}}\right)\right]^{2}
$$

Here $U$ is the local convection speed of the turbulence in the centre shear layer where the ring is placed, $r_{0}$ is the ring radius, $\kappa_{n}=n / r_{0}$ is the azimuthal (spanwise) wavenumber of the incident helical gust or the jet mode of order $n, T$ the projected 
(a)

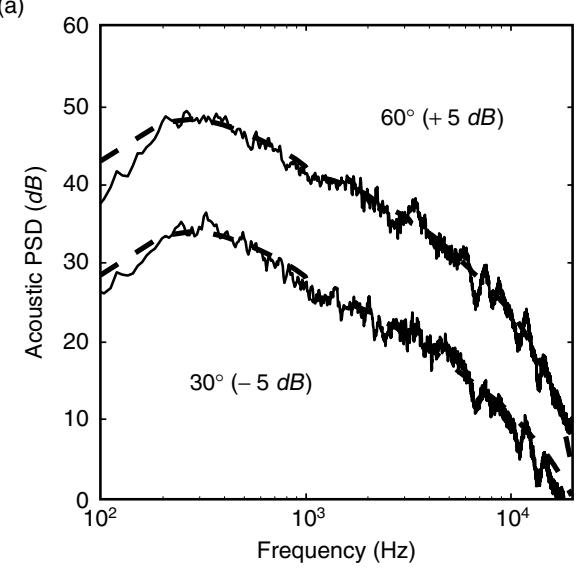

(b)

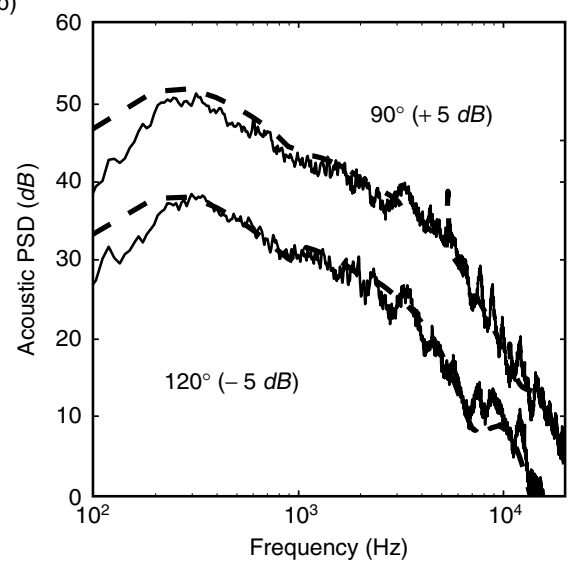

Figure 8: $\quad$ Measured (cont.) versus predicted (dashed) far-field spectra of the turbulence-interaction noise from a flat ring in a circular jet, from [20]. Spectra at different angles from the jet axis, shifted for the sake of clarity. The lower frequencies (below $300 \mathrm{~Hz}$ ) escape the validity range of the assumption of locally quasi-homogeneous and isotropic turbulence.

distance of the observer in the mid-chord plane of the ring. The summation is discrete instead of continuous by virtue of the periodicity condition in cylindrical coordinates.

The very good agreement found when using for $\mathcal{L}_{T I}$ the same expression as in section 2.3.1 for the case of a rectangular airfoil, shown in Fig. 8, indirectly validates the response functions deduced from Schwarzschild's technique for all oblique gusts, both sub- and supercritical. It also proves that the exact solution for the unsteady lift derived in Cartesian coordinates provides a relevant approximate solution of the unsolved problem in cylindrical coordinates. Therefore the underlying assumption is an extension technique to circular geometries and could be used with benefit to investigate other problems, such as the broadband noise generated in a turbofan engine as the turbulent wakes shed from the rotor blades impinge on the edge of the splitter distributing the flow in both the primary and by-pass ducts.

\subsubsection{Effect of the angle of attack}

The effect of the angle of attack on the response to incoming turbulence may be a matter of controversy in view of available experimental or theoretical studies. Some investigations indicate that the effect is weak or negligible for an airfoil in the homogeneous and isotropic turbulence generated by a grid in laboratory experiments, as reported in [10, 29]. Indeed the observed variations are less than $1 d B$. Recent investigations [19] suggest that the wavenumber spectrum for anisotropic turbulence is a key parameter and this an isotropy could induce a significant effect of the angle of attack and of the geometry. 


\subsection{Trailing-edge noise}

Experimental studies of the trailing edge noise require a clean inflow with residual turbulence rates below $1 \%$. The angle of attack is the most crucial parameter and must be adjusted accurately. The background noise of the wind tunnel defined as the noise with the airfoil removed but in the presence of flow over the side-plates, must be as low as possible, because the trailing-egde noise of moderately loaded airfoils is generally weak. Occasionally, at least if the sound is measured directly without resorting to phased-array techniques to isolate the different sources of sound, background-noise subtraction must be performed. The tested airfoil is implemented with flush-mounted wall-pressure sensors, and spanwise and chordwise arrays of sensors are needed close to the trailing-edge.

Most reported works are based on the use of outer boundary-layer variables for the scaling of the wall-pressure spectrum, as

$$
\Psi_{p p}=\frac{\Phi_{p p}}{\pi \rho_{0}^{2} \delta_{1} U_{0}^{3}}
$$

introducing the displacement thickness of the boundary layer $\delta_{1}$ (another thickness scale could be chosen as well). A physically consistent generic form of the function $\Psi$ is reasonably considered as

$$
\Psi_{p p}=\frac{\omega / \omega_{m}}{\left[1+\left(\omega / \omega_{m}\right)^{2}\right]^{3 / 2}} \mathcal{F}\left(\omega / \omega_{c}\right)
$$

where $\omega_{m}$ and $\omega_{c}$ are characteristic frequencies for which the slope of the reduced spectrum changes. The expected slope $\omega^{-2}$ is not always clearly observed but dominates an extended frequency range for airfoils experiencing laminar separation bubbles at the leading edge (results are not shown here). Therefore the expression can be used for dimensional analysis. The function $\mathcal{F}$ reproduces the fast decrease of the spectrum envelope at the highest frequencies, typically like $\omega^{-5}$. It is not believed to significantly contribute to the sound radiation because it corresponds to very small scales. Intuitively, thicker boundary layers correspond to lower frequencies at the same flow speed and for the same flow regime. Therefore the angular frequency can be replaced by $\omega \delta_{1} / U_{c}$ in the model reduced spectrum. Based on these arguments an asymptotic analysis can be proposed for trailing-edge noise in the same way as for turbulence-interaction noise, making use of the high-frequency asymptotic behaviour of $\mathcal{I}$, typically $|\mathcal{I}|^{2} \propto$ $M_{c} /(k c)^{2}$ with $M_{c}=U_{c} / c_{0}$. In this case, the correlation length can be given its expression according to Corcos' model, which generally is consistent at high frequencies: $l_{y} \simeq \delta_{1} /\left(k_{1} \delta_{1}\right)$. This expression again makes use of the fact that the correlation length must be roughly proportional to the boundary layer thickness. Finally the following scaling law is obtained 


$$
\frac{c_{0} S_{0}^{2} S_{p p}}{\rho_{0}^{2} U_{0}^{4} L c^{2}} \propto\left(\frac{x_{3}}{S_{0}}\right)^{2}\left(\frac{\delta_{1}}{c}\right)^{2} \frac{\mathcal{F}\left(\omega / \omega_{c}\right)}{\left(k_{1} \delta_{1}\right)^{3}}
$$

in which the factor $\mathcal{F}$ can be discarded, in the sense that it has the same role as the decay to the Kolmogorov scale often ignored in the model of turbulence velocity spectra for turbulence-interaction noise analysis. In fact this result is quite similar to the high-frequency limit of turbulence-interaction noise, except that the relevant scale is now the boundary-layer thickness instead of the integral length scale of the grid turbulence. It is less convincing because of the large amount of possible types of flows over an airfoil. Indeed very different wall-pressure spectra $\Phi_{p p}$ can be encountered, which correspond to different sets of parameters $\left(\delta_{1}, \omega_{m}, \omega_{c}\right)$. Furthermore, the wallpressure statistics beneath boundary layers in the presence of adverse pressure gradients are better scaled using the inner variables of the boundary layer, or mixed variables [30].

Because of the variety of trailing-edge flows, another way to track general trends is to write down the far-field sound formula (8) as

$$
\Sigma^{*}=\frac{S_{0}^{2} S_{p p}}{L \Phi_{p p y} l}=2\left(\frac{k c}{4 \pi}\right)^{2}\left(\frac{x_{3}}{S_{0}}\right)^{2}|\mathcal{I}|^{2}
$$

This makes $\Sigma^{*}$ a non-dimensional quantity which should not depend on the flow features, all grouped in the factor $\Phi_{p p} l_{y}$. Yet remaining but small differences can arise from the various convection speeds $U_{c}^{y}$ corresponding to different flow regimes, since $U_{c}$ enters as a parameter in the expression of $\mathcal{I}$. A global invariant is finally expected by forming the ratio $\Sigma=\Sigma^{*} /(k c|\mathcal{I}|)^{2}$. This quantity is illustrated in Fig. 9-a, reproduced from [18]. The data from the CD airfoil and the flat plate of section 3.1 tested in the wind tunnel WT1 at different angles of attack corresponding to attached flows are superimposed with the NACA-0012 results of Brooks \& Hodgson [22]. The results collapse reasonably with regard to the very different flow conditions, around the blackline theoretical invariant. It must be noted that remaining discrepancies might be partly attributed to the scattering of airfoil noise by the lip corners of the nozzle. The scattering is more important in the WT1 and is known to introduce artificial dips and humps of a couple of decibels in the spectral shape [23] (no correction is made in the results).

Predictions in dimensional variables are shown in Fig. 9 for the $\mathrm{CD}$ airfoil and compared with the measurements, at three different geometrical angles of attack triggering different flow regimes, all described in [31]. The $-5^{\circ}$ configuration corresponds to the onset of Tollmien-Schlichting (TS) waves on the suction side. The tones resulting from amplification by the acoustic feedback have been removed from the spectrum. Indeed they are correlated over a too large spanwise extent for being covered by the statistical model. The remaining broadband part is very well reproduced by the model, including the shape of the humps, because the primary TS waves are a highly coherent mechanism even when ignoring the feedback. This allows measuring 
(a)

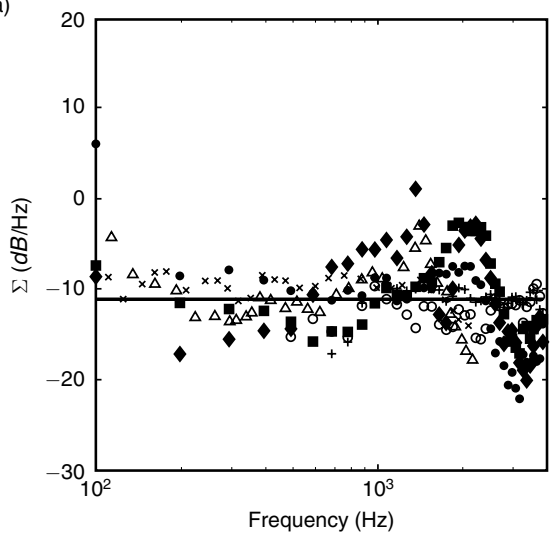

(b)

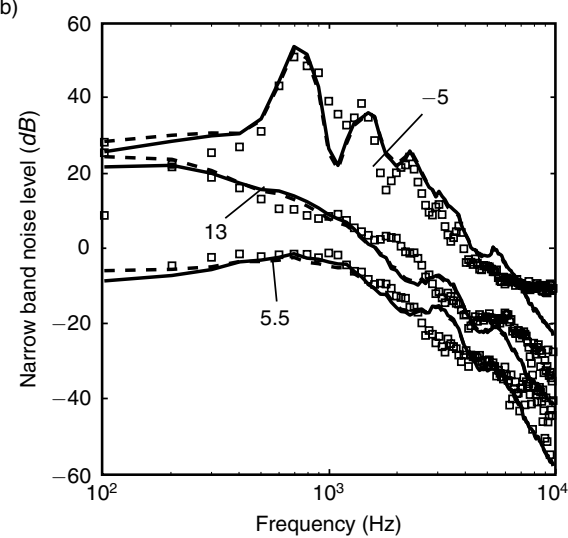

Figure 9: (a): Pseudo-invariant for trailing-edge noise, grouping results for the $\mathrm{CD}$ airfoil (dark symbols), for the flat plate $(x$ and $\Delta$ ) and for a NACA-0012 [22] $(\circ$ and +$)$ in different speed and angle of attack configurations [18]. The black line indicates the theoretical value. (b): measured versus predicted trailing-edge noise spectra from the CD airfoil at different angles of attack. The symbols stand for the measurements and the lines for the predictions using original (dashed) and extended (cont.) Amiet's models. All data refer to the mid-span plane at $90^{\circ}$ to the chord line.

accurately the coherence over an extended frequency range. The other two angles of $5.5^{\circ}$ and $13^{\circ}$ correspond to turbulent boundary layers, with and without leading-edge separation bubbles. In these cases the very low spanwise coherence is much harder to evaluate at high frequencies by means of the spanwise array of wall-pressure probes. Therefore it is deduced from the measurements as far as possible and continued by a fit with theoretical trends, typically a Corcos model or ad hoc models [31]. An overall agreement is obtained, showing that the model is reliable when fed with directly measured input data. The main issue dealing with industrial applications is the difficulty to get the wall-pressure statistics with means other than experimental. At moderate Mach numbers, incompressible LES is the minimum required computational effort [5], but it may not be acceptable. A possible alternative is to resort to a RANS computation to infer the inner and/or outer scales of the boundary layers, and to use it for an evaluation of the spectrum $\Phi_{p p}$, for instance through a model such as eq. (17). This approach is presently incomplete, facing the wide variety of possible configurations encountered in rotating blade technology, because $\Psi$ depends on the loading conditions by the mean-pressure gradient, on the one hand, and because model expressions are still missing for the correlation length $l_{y}$, on the other hand. Typically, plotting $\Psi$ for the set of boundary layers reported in the literature would exibit a large scatter and a global increase of $10 d B$ between zero and adverse pressure gradient configurations. A recent successful attempt for including the pressure gradient in the 
scaling of the wall-pressure spectrum has been reported by Rozenberg et al [30]. It must be considered as a first step still to be confirmed by testing against a wider data base.

\subsection{Vortex-shedding noise}

Vortex-shedding sound is produced in clean inlet flow and moderate loading conditions on plates or airfoils with thick trailing edges, and is more typical of blunt trailing-edges. It has currently a narrow-band signature around a Strouhal number based on the wakeexternal flow speed and the trailing-edge thickness $S_{t}=f h / U_{0} \simeq 0.2$. The peak is wider and reduced in amplitude if the incoming flow from the boundary layers carries turbulent eddies able to make the vortex shedding less coherent. Therefore the vortexshedding noise of a blunted airfoil in clean flow can be heard or not, depending on the angle of attack or the loading conditions, since these conditions determine the boundary layer thickness and turbulence. In the presence of intense incoming turbulence trailingedge noise is not observed anymore.

Vortex-shedding noise signatures in self-noise spectra for a flat plate inclined at different angles are reported in Fig. 10. Results are collected from the two aerodynamic wind tunnels shown in Fig. 3 for which the flow width to chord length ratios are 1.3 (tunnel WT1) and 5 (tunnel WT2). The peak level decreases as the angle of attack increases, whereas in the same time the low-frequency part of the spectrum increases, because trailing-edge noise is also generated. Due to the mean flow deflection induced by the aerodynamic load on the plate at finite angle of attack, which depends on the flow width, different effective loading conditions are achieved at the same non-zero angle of attack (Fig. 10-b). In contrast the plate encounters similar conditions in the WT2 at $7.5^{\circ}$ and in the WT1 at $10^{\circ}$ (Fig. 10-c). It is noted that as the peak is reduced in amplitude, its frequency is shifted towards higher frequencies. This is attributed to the acceleration of the local pressure-side flow around the edge at increasing angle of attack, and differs from the lower-frequency shift observed for instance when putting a rod in a turbulent stream [32].

Predictions using the formulae of section 2.3.3 require the statistics of the near-wake upwash as input data. This information is available either from measurements made on a dedicated setup using for instance cross-wire anemometry, or from a LES of the flow. For bodies of small relative thickness and rectangular trailing edges such as plates, a nice collapse of the velocity spectra can be obtained when plotting the results in reduced variables, as shown by Fig. 10-d for a distance to the edge of $2 h$. Therefore a model velocity spectrum for the peak can be proposed, featured by the blue line, and used for any similar application. The correlation length $l_{y}$ also needed as input data is easily obtained from measurements. In contrast it is hardly deduced from LES because it requires a large $3 \mathrm{D}$ computational domain, typically of a spanwise extent of 6 or $7 \mathrm{~h}$, very demanding in computational resources. This point remains an issue when using the analytical model to post-process incompressible LES fields.

Narrow-band predictions based on 'hybrid' input data are reported and compared to the measurements in Fig. 11. The velocity model of Fig. 10-d is taken as $S_{w w}$ and $l_{y}$ has been deduced from wall-pressure measurements taken closely upstream of the edge. It is argued that the spanwise coherence of that pressure field is similar to the coherence 
(a)

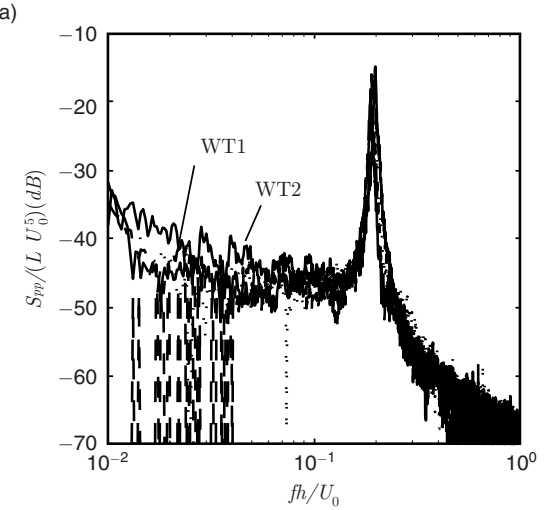

(c)

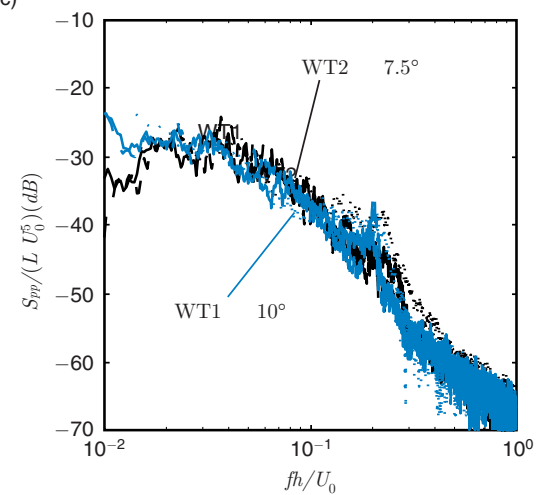

(b)

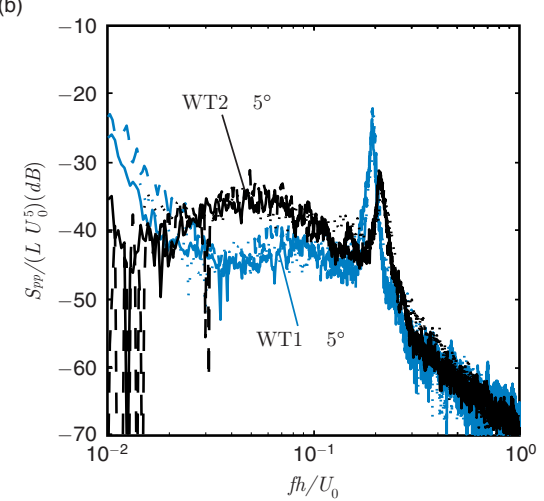

(d)

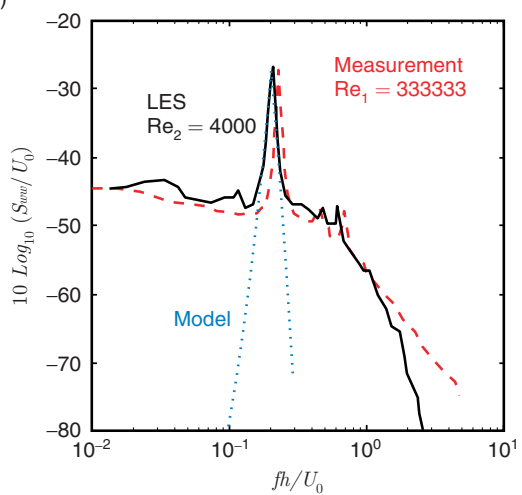

Figure 10: Self-noise spectra for an inclined flat plate of 3\% thickness. Data scaled by $\left(\mathrm{U}_{0}^{5} \mathrm{~L}\right)$. (a) zero angle of attack regime, (b) intermediate regimes, (c) breakdown of the vortex shedding. Vertical lines are artefacts due to subtraction of the background noise. (d): normal velocity spectra in the near-wake at zero angle of attack, from both measurements (red) and LES (black [33]); peak model in blue.

of the near-field upwash velocity. A very good agreement is found at the zero angle of attack for which the vortex-shedding sound is well emerging. At the $5^{\circ}$ angle of attack no velocity measurements were available and LES data are used for $S_{w w}$. But the nozzle flow deflection resulting from the inclination of the plate has not been reproduced in the simulations and an expectedly equivalent higher angle of attack in free stream has been assumed. This leads to some uncertainty in the input data and explains the lower and upper bounds of the predictions. It must be reminded that in this case the flow separation occuring at the leading edge of the inclined plate triggers a turbulent boundary layer which is scattered as sound at the trailing-edge. This trailing-edge noise 


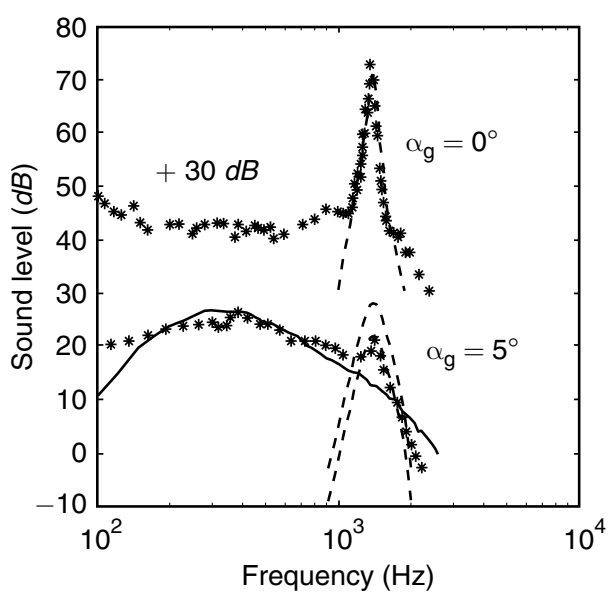

Figure 11: Measured (*) versus predicted (dashed lines) vortex-shedding spectra for an inclined flat plate of $3 \%$ thickness. Upper and lower bounds results at $5^{\circ}$ angle of attack correspond to uncertainty in the input data. The continuous line is the predicted trailing-edge noise contribution.

contribution can be predicted by the model of section 2.3.2 and the prediction is shown as the black line in the figure. It is currently accepted that true TE-noise occurs for a relatively large ratio of boundary layer thickness to trailing-edge thickness $\delta^{\circ} / h$ and VS-noise for small ratios. The present results with the flat plate show that both are observed in the same configuration if the corresponding frequency ranges do not interfere and/or if the rate of turbulence in the boundary layers is low enough to make the formation of wake vortices possible.

The good agreement obtained with the moderately inclined flat plate when using reliable input data directly taken from numerical simulations and experiments suggests that similar configurations can be treated the same way. The model Katana blade investigated recently in [34] is well suited for this. But the compared measured sound spectra of Fig. 12 in reduced variables exhibit large differences, unlike the preceding mechanisms. The high-frequency part of the spectrum for the Katana blade is attributed to Tollmien-Schlichting waves which develop between the ridge and the corner of the back-edge, and which do not take place on the flat plate. The latter has turbulent boundary layers due to the local separation at the leading edge and a much larger chordto-thickness ratio $c / h$ around 33, whereas this ratio is only 6 for the Katana blade. Yet the peaks at the Strouhal frequencies differ significantly. The discrepancies would be found even larger when introducing the chord length as a scaling variable, as made for turbulence-interaction noise. The vortex-shedding flows are not similar in both cases, and the near-wake velocity data of Fig. 10-d cannot be transposed. The cross section of the Katana blade is also much more compact. Since non-compactness is known to induce cancellations by retarded-time differences between chordwise-separated points, it is not suprising that the Katana blade is found specifically louder than the flat plate. 

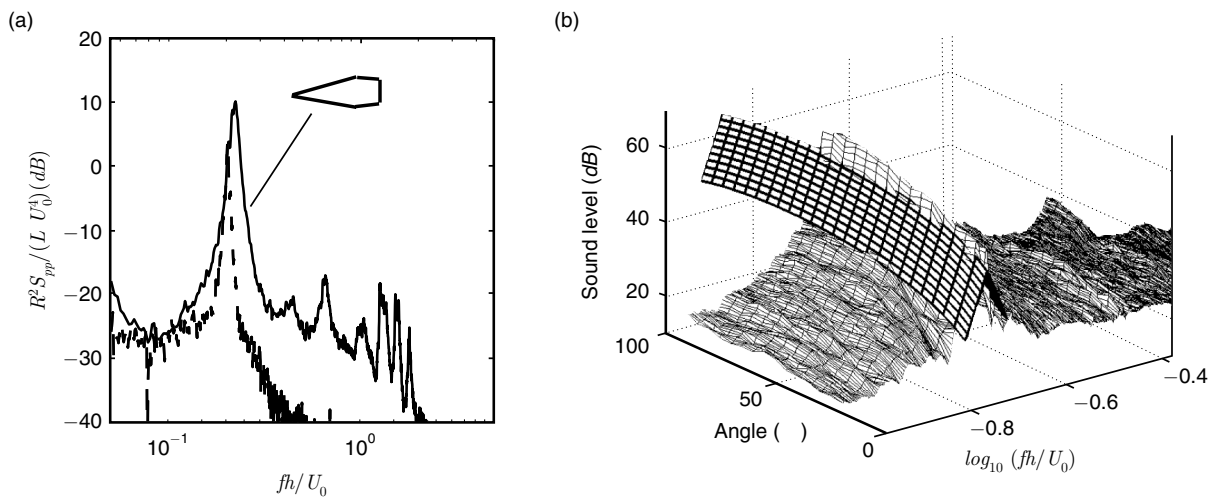

Figure 12: (a) Measured vortex-shedding spectra for a flat plate (dashed blue) and for a Katana blade (black) at zero angle of attack. Failure of the scaling due to different chord lengths and flow features. (b) failure of the vortexshedding noise model applied to the Katana with transposed flat-plate input data.

Furthermore the streamwise mean-pressure gradient over the Katana blade strongly differs from the one over a long flat plate and may lead to different conditions for the onset of the vortex shedding. In the preliminary study of reference [34], the velocity fluctuations have not been measured. When the flat-plate results replace the missing input data for the acoustic model, the predicted sound level is significantly underestimated, as shown in Fig. 12.

\section{EXTENSIONS OF THE ANALYTICAL MODELS}

\subsection{Rotating blade segments and related issues}

Except when dealing with high-lift device noise and related topics, the single-airfoil theories are devoted to subsonic rotating blade noise predictions. Now the relative mean flow and the statistical parameters of the disturbances on a blade vary along the span, from hub to tip. This is why currently the analytical models of previous sections are applied by means of a strip-theory approach. Essentially a blade is split into segments, each of which is attributed a set of aerodynamic parameters. The contributions to the far-field sound are calculated for each segment considered as an isolated airfoil, and summed as uncorrelated. Different issues are revealed in the approach. First the motion of a blade segment relative to the observer is not taken into account in the isolated-airfoil models. It is often introduced as a correction by assuming that the rotating motion is locally equivalent to a tangent translating motion. A correction is applied to account for the Doppler frequencyshift, and an azimuthal average is finally calculated over all angular positions of the blade segment. This simplified approach avoids the repeated calculus of Bessel functions that would be typically needed in an exact formulation, such as the theory developed by Homicz \& George for the noise of a rotor embedded in a turbulent flow [35]. It is justified as far as the characteristic sound frequencies remain high enough when compared to the 
rotational frequency, and holds as well for other broadband-noise mechanisms. Very reliable results have been obtained this way in an investigation of the turbulence-ingestion noise of a rotor reported by Paterson \& Amiet [36]. Another point specific to turbulenceinteraction noise is that the lift fluctuations on successive blades can be correlated if the ingested turbulence is stretched in the axial direction. This occurs in strong suction flows and possibly in some casing boundary-layer flows. In principle the anisotropy of the turbulence should be included at the early steps of the analytical modeling. A simple trick can be used instead, by which the homogeneous and isotropic turbulence model spectrum is distorted along one direction to generate realistic elongated scales [36]. In contrast trailing-edge noise sources are effectively uncorrelated from one blade to another one.

The last and most prominent issue is that the spanwise extent of a blade segment should be much larger than the correlation length $l_{y}$ in principle. This is hardly compatible with the context of rotating blades. More generally the models are derived assuming spanwise homogeneous random processes and their application is questionable in non-homogeneous turbulent flows. Furthermore some artefacts have been reported when using the general formulae (1) and (7), from which one cannot get away a prior $i$ for small segments. The formulae are exact when applied to a rectangular airfoil of arbitrary aspect ratio. As such they are useful for aircraft wings, typically. However if the airfoil is split into smaller spanwise segments, summing the contributions of all segments as uncorrelated sources does not reproduce the noise from the complete airfoil. This is because large spanwise aerodynamic wavenumbers $k_{2}$ are correlated over the entire span when the Fourier expansion in gusts is performed. Cutting the span in segments artificially suppresses this correlation. The error is certainly negligible if the span of each segment remains much larger than the statistical correlation lengths, but it is significant if not. The point is that for a rotating blade discretization, relatively small spanwise segments may be needed to approximately account for the real blade geometry and for the flow variations. An elegant way of overcoming this difficulty for spanwise-varying incident conditions has been proposed by Christophe et al [37]. The idea is to consider a blade segment of limited spanwise extent $L$ as the difference between two overlapping rectangular airfoils of same chord and much larger aspect ratios (span lengths $L_{1}, L_{2} \gg L$, with $L_{2}-L_{1}=L$ ). The segment contribution is expressed as the difference between the two sounds of the overlapping airfoils, both evaluated using the simplified formulae (2) and (8). The large aspect ratio assumption implicitly generates a realistic treatment of the large wavenumbers.

\subsection{Camber corrections}

The assumption of zero camber can be partly released in the case of a moderate camber. Indeed linearized theories of unsteady aerodynamics state that the unsteady lift is distributed over an airfoil as if the airfoil was an ideal flat plate. But the sound radiation itself can be calculated in a second step from the unsteady lift by distributing it along the real mean camber line of the airfoil instead of a straight line. Retarded-time differences or phase shifts are induced this way and possibly modify the radiated field. Such a refinement requires that the radiation integral $\mathcal{L}$ or $\mathcal{I}$ be computed numerically instead of being derived analytically. It has been tested for turbulence-interaction noise 
[26] and similar corrections are possible for trailing-edge noise. Essentially the effect of the curvature is to distort the symmetric zero-camber directivity pattern with respect to the chord line, more sound being radiated in the half-space facing the pressure side and less sound in the opposite half-space. The asymmetry is more pronounced at high frequencies. The correction is not expected to have a strong effect for the moderate camber angles encountered in compressors, fans and turbofans, and typically induces a noise asymmetry of the order of $1 d B$.

\subsection{Skewed gusts}

In some cases, such as an aircraft swept wing or the swept blades of an advanced fan, the mean flow velocity is not perpendicular to the spanwise direction, so that the corresponding convected Helmholtz equation includes an additional term. As long as the span is kept infinite for the sake of calculating the distributed equivalent dipoles, Schwarzschild's technique remains applicable, again by means of a suited change of variables. This is essentially because the boundary conditions are still defined uniformly along the span and can be decomposed by successive half-planes for the solving procedure. In fact the corresponding formulae for the lift induced by the impingement of upstream turbulence on a swept plate have been derived by Adamczyk [38] using the Wiener-Hopf technique, which is mathematically equivalent. The next point is that when calculating the sound field from the unsteady lift the actual shape of the plate or of the blade segment is not rectangular anymore but rather trapezoidal. Indeed in the case of axial rotors the strip-theory generates cuts along the direction of the incident mean flow in the tangential direction. Another proper change of variables still makes the integration tractable analytically on a trapezoidal segment. Therefore skewness is not a limitation of the technique.

Other issues are related to the convection speed of boundary-layer disturbances when addressing the trailing-edge noise of a fan with swept blades. Indeed the convection speed and the mean flow speed out of the boundary layer may not be in the same direction. This is presently a stumbling block in the practical application of the analytical formulae. Yet some aspects of swept trailing edges are addressed for instance by Howe [39].

\subsection{Tip effects}

Other simple extensions of the analytical models can be proposed on the same Schwarzschild's technique. One of them is based on the assumption of an imaginary or complex spanwise wavenumber $k_{2}$, acceptable as a possible solution of the Helmholtz equation. Its physical meaning is a concentration of the incident disturbances at some spanwise location with an exponential decay away from that point. Even though localized random flows may be incompatible with the necessary condition of statistical homogeneity, a closed-form solution of the trace of the field on the surface can be derived. A recent typical application to the tip-clearance noise from a ducted fan blade is discussed in reference [40]. Some part of that noise is attributed to the formation of a tip vortex which is swept past the tip corner of the trailing edge and is interpreted as specific, concentrated trailing-edge noise sources. 


\section{CONCLUDING REMARKS}

In view of all reported results, Schwarzschild-based models are very reliable when applied to airfoil shapes which remain close to the classical assumptions of the linearized theory of unsteady aerodynamics. This includes small thickness, camber and angles of attack, as well as small disturbances. They apply for any subsonic Mach number and account for the non compactness. Dealing with turbulence-interaction noise, the assumptions hold in practice for thicknesses below a few percent of the chord length, total cambers up to 20 to $25^{\circ}$, and incoming disturbance rates lower than $10 \%$. Amiet's model may overestimate the turbulence-interaction noise of thick airfoils by up to $10 d B$ or more at high frequencies at low speeds. Therefore it is accurate for thin compressor blades at high subsonic speeds but may require corrections for low-speed fans. Empirical corrections can be applied, based on the incoming flow speed, the turbulence integral length scale and the leading-edge thickness. The input data can be easily obtained from RANS computations, currently performed by manufacturers of rotating blade technologies, assuming homogeneous and isotropic turbulence. Yet accuracy issues remain for anisotropic turbulence.

The trailing-edge noise model can be very accurate when fed with directly measured wall-pressure data, but its use as a design tool in industrial context in connection with RANS simulations must still be refined. Uncertainties will remain within a range of $10 d B$ for boundary layers in adverse pressure gradients, until the relationship between the wall-pressure statistics and the mean-flow parameters currently investigated will be validated over an extended data base. Vortex-shedding noise is another mechanism that has been modeled using Schwarzschild's technique. The correponding solutions are accurate for rectangular edges of lightly loaded blades with large chord-to-thickness ratios. They might be harder to use in more general configurations, because the velocity statistics in the near-wake needed as input data strongly depends on the loading conditions and remains a challenging task for CFD tools.

Globally the validation of the models on single airfoils makes them reliable for application to rotating blades, by means of a spanwise segment splitting. Yet numerical tests not detailed in the paper have shown that the exact formulations for arbitrary aspect ratios are well suited for rectangular airfoils in translating motion with respect to the air, but that the large aspect ratio approximations are surprisingly more suited to a striptheory treatment of rotating blades, provided that the 'overlapping rectangles' jack-knife described in the text is included. It is believed that the class of mathematical problems based on Schwarzschild's technique still has possible extensions in the aeroacoustics of subsonic blades, typically addressing swept blades and tip effects. Some of them are presently in progress.

\section{REFERENCES}

[1] J.E. Ffowcs Williams \& D.L. Hawkings, "Sound generation by turbulence and surfaces in arbitrary motion", Phil. Trans. Roy. Soc., A 264, 1969. 
[2] J.E. Ffowcs Williams \& L.H. Hall, "Aerodynamic sound generation by turbulent flow in the vicinity of a scattering half-plane", J. Fluid Mech., 40, pp. 657-670, 1970.

[3] W.K. Blake, "Mechanics of flow-induced sound and vibration", Academic Press, Orlando, 1986.

[4] M.S. Howe, "A review of the theory of trailing-edge noise", J. Sound \& Vib. 61 (3), pp. 437-465, 1978.

[5] M. Wang, S. Moreau, G. Iaccarino \& M. Roger, "LES prediction of wall-pressure fluctuations and noise of a low-speed airfoil", Int. J. of Aeroacoustics, 8 (3), pp. 177-198, 2009.

[6] S.A.L. Glegg, "The response of a swept blade row to a three-dimensional gust", J. Sound Vib. 227 (1), pp. 29-64, 1999.

[7] H. Posson \& M. Roger, "Parametric Study of Gust Scattering and Sound Transmission Through a Blade Row", $13^{\text {th }}$ AIAA/CEAS Aeroacoustics Conference Rome, paper 2007-3690, 2007.

[8] M. Landahl, “Unsteady transonic flows”, Pergamon Press, 1961.

[9] R.K. Amiet, "Acoustic radiation from an airfoil in a turbulent stream", J. Sound \& Vib. 41, pp. 407-420, 1975.

[10] R.W Paterson \& R.K. Amiet, "Acoustic radiation and surface pressure characteristiccs of an airfoil due to incident turbulence", NASA CR 2733, 1976.

[11] R.K. Amiet, "High-frequency thin-airfoil theory for subsonic flow", AIAA $J$. 14(8), pp. 1076-1082, 1976.

[12] M. Roger \& S. Moreau, "Back-scattering correction and further extensions of Amiet's trailing-edge noise model; part I: theory", J. Sound \& Vib. 286, pp. 477-506, 2005.

[13] M. Roger, S. Moreau \& A. Guédel, "Vortex-Shedding Noise and PotentialInteraction Noise Modeling by a Reversed Sears' Problem", $12^{\text {th }}$ AIAA/CEAS Aeroacoustics Conference, Cambridge MA, paper 2006-2607, 2006.

[14] D.P. Lockard \& P.J. Morris, "A parallel implementation of a computational aeroacoustic algorithm for airfoil noise", J. Computational Acoustics, 5 (4), pp. 337-353, 1997.

[15] M.S. Howe, "Edge-source acoustic Green's function for an airfoil of arbitrary chord, with application to trailing-edge noise", Quart. J. Mech. Appl. Math., 54 (1), pp. 139-155, 2001.

[16] R.K. Amiet, "Noise due to turbulent flow past a trailing-edge", J. Sound \& Vib., 47 (3), pp. 387-393, 1976.

[17] Q. Zhou \& P. Joseph, "A frequency domain numerical method for airfoil broadband selfnoise prediction" J. Sound \& Vib. 299, pp. 504-519, 2007.

[18] S. Moreau \& M. Roger, "Back-scattering correction and further extensions of Amiet's trailing-edge noise model; part II: applications", J. Sound \& Vib. 323, pp. 397-425, 2009. 
[19] P. F. Mish \& W. J. Devenport, "An experimental investigation of unsteady surface pressure on an airfoil in turbulence - Part 1: effects of mean loading", J. Sound \& Vib., 296 (3), pp. 417-446, 2006.

[20] M. Roger \& S. Serafini, "Interaction noise from a thin annulus in a circular jet", $11^{\text {th }}$ AIAA/CEAS Aeroacoustics Conference, paper 2005-2958, Monterey 2005.

[21] S. Moreau, M. Henner, G. Iaccarino, M. Wang \& M. Roger, "Analysis of flow conditions in free-jet experiments for studying airfoil selfnoise", AIAA Journal $4 \mathbf{1}$ (10), pp. 1895-1905, 2003.

[22] T.F. Brooks \& T.H. Hodgson, "Trailing-edge noise prediction from measured surface pressures", J. Sound \& Vib., 78 (1), pp. 69-117, 1981.

[23] S. Moreau, C. Schram \& M. Roger, "Diffraction effects on the trailing-edge noise measured in an open-jet anechoic wind tunnel", $13^{\text {th }}$ AIAA/CEAS Aeroacoustics Conference, paper 2007-3409, Rome, 2007.

[24] S. Oerlemans \& P. Migliore, "Aeroacoustic Wind-Tunnel Test of Wind-Turbine Airfoils", $10^{\text {th }}$ AIAA/CEAS Aeroacoustics Conference, paper 2004-3042, Manchester, 2004 .

[25] J.K. Staubs, W.J. Devenport \& S.A.L. Glegg, "Sound Radiation from a Series of Airfoils Immersed in Grid-Generated Turbulence", 14 $4^{\text {th }}$ AIAA/CEAS Aeroacoustics Conference, paper 2008-3018, Vancouver 2008.

[26] S. Moreau, M. Roger \& V. Jurdic, "Effect of angle of attack and airfoil shape on turbulence-interaction noise", $11^{\text {th }}$ AIAA/CEAS Aeroacoustics Conference, paper 2005-2973, Monterey, 2005.

[27] W.A. Olsen, "Noise generated by impingement of turbulent flow on airfoils of varied chord, cylinders, and other flow obstructions", NASA TM X-73464, 1976.

[28] J. Gershfeld, "Leading edge noise from thick from thick foils in turbulent flows", J. Acoust. Soc. Amer 116, pp. 1416-1426, 2004.

[29] S. Moreau \& M. Roger, "Competing broadband noise mechanisms in low-speed axial fans", AIAA Journal, 45(1), pp. 48-57, 2007.

[30] Y. Rozenberg, M. Roger \& S. Moreau, "Fan blade trailing-edge noise prediction using RANS simulations", EuroNoise - Acoustics'08, paper 4pNSq2, Paris, 2008.

[31] M. Roger \& S. Moreau, "Broadband self-noise from loaded fan blades", AIAA Journal 42 (3), pp. 536-544, 2004.

[32] M.H. Ross, D.W. Shannon \& S.C. Morris, "Unsteady lift and radiated sound generated by a circular cylinder in a single stream shear layer", $14^{\text {th }}$ AIAA/CEAS Aeroacoustics Conference, paper 2008-2962, Vancouver 2008.

[33] K. Chang, J. Seo, Y. Moon \& M. Roger, "Prediction of flat-plate self-noise", $12^{\text {th }}$ AIAA/CEAS Aeroacoustic Conference, AIAA paper 2006-2513, Cambridge MA, 2006.

[34] M. Roger, "Coupled oscillations in the aeroacoustics of a Katana blade", EuroNoise - Acoustics'08, paper 1pNSa12, Paris, 2008. 
[35] G.F. Homicz \& A.R. George, "Broadband and discrete frequency radiation from subsonic rotors", J. Sound \& Vib., 36 (2), pp. 151-177, 1974.

[36] R.W Paterson \& R.K. Amiet, "Noise of a model helicopter rotor due to ingestion of turbulence", NASA CR 3213, 1979.

[37] J. Christophe, J. Anthoine, P. Rambaud \& S. Moreau, "Numerical issues in the application of an Amiet model for spanwise-varying incoming turbulence", $14^{\text {th }}$ AIAA/CEAS Aeroacoustics Conference, paper 2008-2865, Vancouver 2008.

[38] J.J. Adamczyk, "The passage of an infinite swept airfoil through an oblique gust", J. of Aircraft 11, pp. 281-287, 1974. Also NASA CR 2395, 1974.

[39] M.S. Howe, "Noise produced by a sawtooth trailing edge", J. Acoust. Soc. Amer., 90 (1), pp. 482-487, 1991.

[40] J. Grilliat, E. Jondeau, M. Jacob \& M. Roger, "Broadband noise prediction models and measurements of tip leakage flows", $14^{\text {th }}$ AIAA/CEAS Aeroacoustics Conference, Vancouver, Canada, AIAA Paper 2008-2845, 2008. 
